\title{
Super-Eddington accretion on to the neutron star NGC 7793 P13: Broad-band X-ray spectroscopy and ultraluminous $X$-ray sources
}

\author{
D. J. Walton, ${ }^{1 \star}$ F. Fürst,${ }^{2}$ F. A. Harrison, ${ }^{3}$ D. Stern, ${ }^{4}$ M. Bachetti, ${ }^{5}$ D. Barret,${ }^{6,7}$ \\ M. Brightman, ${ }^{3}$ A. C. Fabian, ${ }^{1}$ M. J. Middleton, ${ }^{8}$ A. Ptak ${ }^{9}$ and L. Tao ${ }^{3}$ \\ ${ }^{1}$ Institute of Astronomy, Cambridge University, Madingley Road, Cambridge CB3 OHA, UK \\ ${ }^{2}$ European Space Astronomy Centre (ESAC), Science Operations Departement, E-28692 Villanueva de la Cañada, Madrid, Spain \\ ${ }^{3}$ Space Radiation Laboratory, California Institute of Technology, Pasadena, CA 91125, USA \\ ${ }^{4}$ Jet Propulsion Laboratory, California Institute of Technology, Pasadena, CA 91109, USA \\ ${ }^{5}$ INAF/Osservatorio Astronomico di Cagliari, via della Scienza 5, I-09047 Selargius (CA), Italy \\ ${ }^{6}$ Université de Toulouse, UPS-OMP, IRAP, F-31028 Toulouse, France \\ ${ }^{7}$ CNRS, IRAP, 9 Av. colonel Roche, BP 44346, F-31028 Toulouse cedex 4, France \\ ${ }^{8}$ Department of Physics and Astronomy, University of Southampton, Highfield, Southampton SO17 1BJ, UK \\ ${ }^{9}$ NASA Goddard Space Flight Center, Greenbelt, MD 20771, USA
}

Accepted 2017 October 9. Received 2017 October 9; in original form 2017 May 29

\begin{abstract}
We present a detailed, broad-band X-ray spectral analysis of the ultraluminous X-ray source (ULX) pulsar NGC 7793 P13, a known super-Eddington source, utilizing data from the XMMNewton, NuSTAR and Chandra observatories. The broad-band XMM-Newton+NuSTAR spectrum of P13 is qualitatively similar to the rest of the ULX sample with broad-band coverage, suggesting that additional ULXs in the known population may host neutron star accretors. Through time-averaged, phase-resolved and multi-epoch studies, we find that two non-pulsed thermal blackbody components with temperatures $\sim 0.5$ and $1.5 \mathrm{keV}$ are required to fit the data below $10 \mathrm{keV}$, in addition to a third continuum component which extends to higher energies and is associated with the pulsed emission from the accretion column. The characteristic radii of the thermal components appear to be comparable, and are too large to be associated with the neutron star itself, so the need for two components likely indicates the accretion flow outside the magnetosphere is complex. We suggest a scenario in which the thick inner disc expected for super-Eddington accretion begins to form, but is terminated by the neutron star's magnetic field soon after its onset, implying a limit of $B \lesssim 6 \times 10^{12} \mathrm{G}$ for the dipolar component of the central neutron star's magnetic field. Evidence of similar termination of the disc in other sources may offer a further means of identifying additional neutron star ULXs. Finally, we examine the spectrum exhibited by P13 during one of its unusual 'off' states. These data require both a hard power-law component, suggesting residual accretion on to the neutron star, and emission from a thermal plasma, which we argue is likely associated with the P13 system.
\end{abstract}

Key words: stars: neutron - X-rays: binaries - X-rays: individual: NGC 7793 P13.

\section{INTRODUCTION}

The ultraluminous X-ray source $\left(\mathrm{ULX}^{1}\right)$ NGC 7793 P13 (hereafter P13), which reaches luminosities of $L_{\mathrm{X} \text {, peak }} \sim 10^{40} \mathrm{erg} \mathrm{s}^{-1}$, was recently found to be powered by an accreting neutron star through the detection of coherent X-ray pulsations (Fürst et al. 2016b; Israel et al. 2017b). Along with M82 X-2, the first ULX pulsar

\footnotetext{
*E-mail: dwalton@ srl.caltech.edu

${ }^{1}$ Here, we define a ULX to be an off-nuclear X-ray point source with a luminosity in excess of $10^{39} \mathrm{erg} \mathrm{s}^{-1}$ in the $0.3-10.0 \mathrm{keV}$ bandpass, assuming isotropy.
}

discovered ( $L_{\mathrm{X} \text {, peak }} \sim 2 \times 10^{40} \mathrm{erg} \mathrm{s}^{-1}$; Bachetti et al. 2014) and NGC 5907 ULX $\left(L_{X}\right.$, peak $\sim 9 \times 10^{40} \mathrm{erg} \mathrm{s}^{-1}$; Fürst et al. 2017; Israel et al. 2017a), only three neutron star ULXs with such extreme luminosities are currently known. These systems are of great interest, as they offer a rare opportunity to study accretion in the highly super-Eddington regime (the Eddington limit for a standard $1.4 \mathrm{M}_{\odot}$ neutron star is $L_{\mathrm{E}} \sim 2 \times 10^{38} \mathrm{erg} \mathrm{s}^{-1}$ ).

There are several observational similarities seen between the P13, M82 X-2 and NGC 5907 ULX systems. All three have broadly similar pulse periods, of order $\sim 1$ s (Bachetti et al. 2014; Fürst et al. 2016b; Israel et al. 2017a,b), and exhibit strong secular spinup owing to the large accretion torques related to their extreme accretion rates; over just $\sim 10 \mathrm{yr}$, the pulse period in NGC 5907 ULX 
evolved from $\sim 1.4$ to $\sim 1.1 \mathrm{~s}$. The pulse profiles observed are also similar, with all three sources showing broad, near-sinusoidal variations. Furthermore, all three show long-term periodicities $(\sim 60$ 80 d; Motch et al. 2014; Kong et al. 2016; Walton et al. 2016c; Hu et al. 2017; Brightman et al., submitted). In the cases of M82 X-2 and NGC 5907 ULX these periods are known to be super-orbital, as variations in their pulse periods have also revealed orbital periods of a few days. No such independent constraints on the orbit are currently available for $\mathrm{P} 13$, where the nature of the $\sim 64 \mathrm{~d}$ period remains uncertain, although it does seem to show some 'jitter' (Motch et al. 2014) that may be indicative of superorbital variations. Finally, all three sources show unusual 'off-states', where the X-ray flux is diminished by a factor of $\sim 50$ or more (e.g. Motch et al. 2014; Walton et al. 2015a; Brightman et al. 2016a). Tsygankov et al. (2016) suggest that these extreme flux modulations may be related to the propeller effect, but their nature is not currently well established, and may even differ between systems.

These systems pose key questions, particularly with regards to how these neutron stars are able to reach such extreme apparent luminosities. The magnetic field of the central neutron star will channel the accretion flow into columns inside the magnetospheric radius ( $R_{\mathrm{M}}$, the point at which magnetic pressure dominates), allowing material to accrete on to the magnetic poles while radiation escapes from the sides of the column (Basko \& Sunyaev 1976). This introduces anisotropy into the radiation field (which is required in order for X-ray pulsations to be observed), and in turn a beaming correction to simple luminosity estimates becomes necessary. However, while some anisotropy is clearly required, it is difficult to explain the near-sinusoidal pulse profiles observed from all three systems in the context of strong beaming of sub-Eddington accretion on to an otherwise standard neutron star (which would require beaming by factors of $\sim 100$ or more).

Some authors have invoked strong, magnetar-level magnetic fields $\left(B \sim 10^{13-14} \mathrm{G}\right.$; e.g. Dall'Osso, Perna \& Stella 2015; Ekşi et al. 2015; Mushtukov et al. 2015). This reduces the scattering cross-section for electrons (Herold 1979), reducing the radiation pressure and in turn increasing the effective Eddington luminosity. However, other authors have instead suggested that the magnetic field is much lower (potentially as low as $B \sim 10^{9} \mathrm{G}$ ) based on the ratio of the spin-up rate to the luminosity, which is an order of magnitude lower than typical X-ray pulsars (e.g. Kluźniak \& Lasota 2015). These authors argue that a disc truncated at a large radius (close to the co-rotation radius, $R_{\mathrm{co}}$, as would occur for a high-B field system; $R_{\mathrm{co}}$ is the point at which the material in the disc co-rotates with the neutron star, and $R_{\mathrm{M}}<R_{\mathrm{co}}$ is required for strong accretion to occur) would not provide the required lever arm to power the observed spin-up. In such a scenario, the extreme luminosities would need to be produced by a highly super-Eddington accretion disc that extends close to the accretor, similar to superEddington accretion on to a black hole (King \& Lasota 2016).

P13 provides a key laboratory for understanding these extreme neutron stars and their link to the broader ULX population. With only three examples, the sample of ULX pulsars is still extremely limited, and there are obstacles to undertaking detailed analyses with M82 X-2 and NGC 5907 ULX. M82 X-2 is heavily confused with its more luminous neighbour M82 X-1 (separated by $\sim 5 \mathrm{arcsec}$ ), and NGC 5907 ULX is situated at a large distance $(\sim 17 \mathrm{Mpc}$; Tully, Courtois \& Sorce 2016) so its observed flux is relatively low. Both sources are also heavily absorbed $\left(N_{\mathrm{H}} \sim 10^{22} \mathrm{~cm}^{-2}\right)$, severely complicating comparisons with other ULXs. In contrast, P13 is both well isolated and relatively unobscured $\left(N_{\mathrm{H}} \sim 10^{21} \mathrm{~cm}^{-2}\right)$. Furthermore, pulsations are only intermittently detected in M82 X-2 and
Table 1. Details of the X-ray observations of NGC 7793 P13 considered in this work.

\begin{tabular}{lcccc}
\hline Epoch & Mission(s) & OBSID(s) & $\begin{array}{c}\text { Start } \\
\text { date }\end{array}$ & $\begin{array}{c}\text { Exposure }^{a} \\
(\mathrm{ks})\end{array}$ \\
\hline XN1 & NuSTAR & $\begin{array}{c}\text { 80201010 } \\
\text { XM-Newton } \\
\text { 0781800101 } \\
\text { Archival data }\end{array}$ & $2016-05-20$ & 115 \\
C1 & Chandra & 3954 & $28 / 46$ \\
C2 & Chandra & 14231 & $2003-09-06$ & 49 \\
C3 & Chandra & 13439 & $2011-12-25$ & 59 \\
C4 & Chandra & 14378 & $2011-12-30$ & 25 \\
X1 & XMM-Newton & 0693760101 & $2012-05-14$ & $26 / 36$ \\
X2 & XMM-Newton & 0693760401 & $2013-11-25$ & $41 / 47$ \\
X3 & XMM-Newton & 0748390901 & $2014-12-10$ & $42 / 48$ \\
\hline Note ${ }^{a}$ XMM-Newton exposures are listed for the EPIC-pn/MOS detectors.
\end{tabular}

Note. ${ }^{a}$ XMM-Newton exposures are listed for the EPIC-pn/MOS detectors.

NGC 5907 ULX (Bachetti et al. 2014; Israel et al. 2017a), while for P13 they have always been detected when the source has been observed at high flux and with sufficient temporal resolution.

In this paper, we present results from the 2016 broad-band observation of P13 with XMM-Newton (Jansen et al. 2001) and NuSTAR (Harrison et al. 2013). Our initial work on these data, presented in Fürst et al. (2016b), focused on the detection and evolution of the Xray pulsations, and here we focus on undertaking a comprehensive spectral analysis. We describe the observations and data reduction in Section 2, and we provide details on our spectral analysis in Sections 3 and 4. We draw comparisons with the broader ULX population in Section 5, and in Section 6 we discuss the results. Finally, we summarize our conclusions in Section 7. Throughout this work, we adopt a distance to NGC 7793 of 3.5 Mpc (Pietrzyński et al. 2010).

\section{OBSERVATIONS AND DATA REDUCTION}

As discussed in Fürst et al. (2016b), the coordinated XMMNewton + NuSTAR observation of P13 that is the focus of much of this work was triggered following the detection of a high flux with Swift (Gehrels et al. 2004), and currently represents the highest flux probed with high signal-to-noise $(\mathrm{S} / \mathrm{N})$ observations for this source to date. In addition to these data, we also consider the archival XMM-Newton and Chandra (Weisskopf et al. 2002) exposures of P13. Details of all the observations considered in this work are given in Table 1. Our data reduction procedure largely follows that outlined in Fürst et al. (2016b), but here we repeat the salient points for clarity.

\subsection{NUSTAR}

The NUSTAR data were reduced using the NUSTAR Data Analysis Software (NUSTARDAS; v1.6.0) following standard procedures; NUSTAR caldb v20160824 was used throughout. We cleaned the unfiltered event files with NUPIPELINE, applying the standard depth correction (which significantly reduces the internal background) and removing passages through the South Atlantic Anomaly. Barycentre corrections were applied to the photon arrival times in the cleaned event files using the DE200 reference frame, and based on the pulse period reported in Fürst et al. (2016b) we phase-stamp each of the events. Source products and instrumental response files were extracted from circular regions of radius 70 arcsec for both of the focal plane modules (FPMA/B) with NUPRODUCTS, with background 
estimated from a much larger region on the same detector as P13. In addition to the standard 'science' data, to maximize the $\mathrm{S} / \mathrm{N}$ we also extract the 'spacecraft science' data following the procedure outlined in Walton et al. (2016b), which provides $\sim 10$ per cent of the good exposure in this case.

\subsection{XMM-Newton}

The XMM-Newton data were reduced with the XMM-Newton Science Analysis System (v15.0.0), following the standard data reduction guide. ${ }^{2}$ The raw data files were cleaned using EPCHAIN and EMCHAIN for the EPIC-pn (Strüder et al. 2001) and each of the EPIC-MOS (Turner et al. 2001) detectors, respectively. As with the NuSTAR data, barycentre corrections were applied to the photon arrival times in the cleaned event files for EPIC-pn using the DE200 reference frame, and we phase-stamp events based on the pulse periods reported in Fürst et al. (2016b); unfortunately the EPIC-MOS detectors do not have sufficient temporal capabilities to resolve the $\sim 0.4$ s pulse period. Source products were typically extracted from circular regions of radius $\sim 40$ arcsec and background was estimated from larger areas on the same $\mathrm{CCD}$ free of other contaminating point sources. The exception was OBSID 0693760101, during which the source was extremely faint (but still clearly detected), so a radius of 20 arcsec was used. As recommended, we only use single-double patterned events for EPIC-pn and single-quadruple patterned events for EPIC-MOS, and we also excluded periods of high background as standard. Instrumental redistribution matrices and ancillary response files were generated for each of the detectors with RMFGEN and ARFGEN, respectively. Finally, after checking their consistency, spectra from the two EPIC-MOS detectors were combined using ADDASCASPEC.

\subsection{Chandra}

The Chandra data were reduced with the CIAO software package (v4.7) following standard procedure. We extracted spectra from the ACIS-S detector (Garmire et al. 2003) level 2 event files and computed instrumental response files using SPECEXTRACT. Although the three 2011 observations all occurred during the extended off-state seen from P13, the source is still clearly detected in all observations. Source spectra are extracted from circular regions of radius 4-5 arcsec, and as with both NUSTAR and XMM-Newton background was estimated from larger areas on the same CCD free of other contaminating point sources. All the observations were performed in the Timed Exposure mode with fixed frame times of $\sim 3.2 \mathrm{~s}$; the time resolution of these Chandra observations is therefore too coarse to detect the $\sim 0.4 \mathrm{~s}$ pulsations from $\mathrm{P} 13$.

\section{NGC 7793 P13: THE BROAD-BAND 2016 DATA SET}

We begin our analysis by focusing on the broad-band XMM-Newton+NuSTAR data set obtained in 2016 (i.e. epoch $\mathrm{XN1}$ ), as these observations have provided the first ever hard Xray $(>10 \mathrm{keV})$ detection of P13. Model fits are performed with XSPEC V12.6.0f (Arnaud 1996), parameter uncertainties are quoted at the 90 percent confidence level for one interesting parameter ( $\Delta \chi^{2}=2.7$, with all other free parameters allowed to vary during the

\footnotetext{
${ }^{2}$ http://xmm.esac.esa.int/
}

error estimation). All models considered include a Galactic absorption component with a fixed column of $N_{\mathrm{H}, \mathrm{Gal}}=1.2 \times 10^{20} \mathrm{~cm}^{-2}$ (Kalberla et al. 2005), and we also allow for absorption intrinsic to the source at the redshift of NGC $7793\left(N_{\mathrm{H} ; \text { int }} ; z=0.000767\right)$. Both neutral absorption components are modelled with the TBNEW absorption code. As recommended for TBNEW, we use the cross-sections of Verner et al. (1996) and the abundance set presented by Wilms, Allen \& McCray (2000). We also allow for cross-calibration uncertainties between the different detectors by including multiplicative constants that are allowed to float between the data sets, fixing EPIC-pn at unity. These constants are always within 10 per cent of unity, as expected (Madsen et al. 2015). Unless stated otherwise, spectra are grouped to have a minimum of 50 counts per energy bin throughout this work in order to facilitate the use of $\chi^{2}$ minimization during our analysis.

\subsection{Time-averaged spectroscopy}

The time-averaged broad-band spectrum observed from epoch XN1 is shown in Fig. 1 (left-hand panel); P13 is detected by NuSTAR up to $\sim 30 \mathrm{keV}$. The broad-band spectrum is qualitatively similar to the broad-band spectra seen from the rest of the ULX population to date (see Section 5). As noted by Motch et al. (2014) and Pintore et al. (2017), who present spectral analyses of the archival $X M M-$ Newton observations of P13, the spectrum below $10 \mathrm{keV}$ requires two broad-band continuum components: a thermal component that contributes below $\sim 2 \mathrm{keV}$, and a harder component that dominates at higher energies. The prior XMM-Newton observations analysed by these authors indicate this higher energy component shows curvature in the $\sim 5-10 \mathrm{keV}$ band, similar to the rest of the ULX population (e.g. Stobbart, Roberts \& Wilms 2006; Gladstone, Roberts \& Done 2009). The NUSTAR data clearly and robustly confirms the presence of this curvature, demonstrating that, as with the rest of the ULX sample observed by NUSTAR to date, the emission that dominates the $2-10 \mathrm{keV}$ band is not a high-energy power-law tail, and that the curvature seen in the XMM-Newton data does mark the start of a high-energy spectral cutoff.

Before undertaking a more detailed analysis of the spectral properties of P13, we fit the average spectrum with a set of models typically applied to other ULXs in the literature to provide a simple comparison. Based on the previous analyses of the archival P13 data, and our prior work on broad-band observations of ULXs with NuSTAR, we apply a set of 2-component continuum models commonly applied to ULX spectra. We start with a model consisting of two accretion disc components, combining the DISKBB and DISKPBB models (Mitsuda et al. 1984; Mineshige et al. 1994). The former is based on the thin accretion disc model of Shakura \& Sunyaev (1973), which has a radial temperature index of $p=3 / 4$ (where $\left.T(r) \propto r^{-p}\right)$ and just has the disc temperature $\left(T_{\text {in }}\right)$ as a free parameter, while the latter also allows $p$ to be varied as a free parameter. Secondly, we also apply a model combining DISKBB with COMPTT (Titarchuk 1994), a thermal Comptonization model which is primarily characterized by the optical depth $(\tau)$ and the temperature $\left(k T_{\mathrm{e}}\right)$ of the scattering electrons (for convenience, we assume the temperature of the seed photons to be that of the DISKBB component, as in previous works). Both of these models provide reasonable fits to the data below $\sim 10-15 \mathrm{keV}$. However, as can clearly be seen in the data/model ratios shown in Fig. 1 (right-hand panels), both models leave a clear excess in the NuSTAR data at the highest energies probed. 

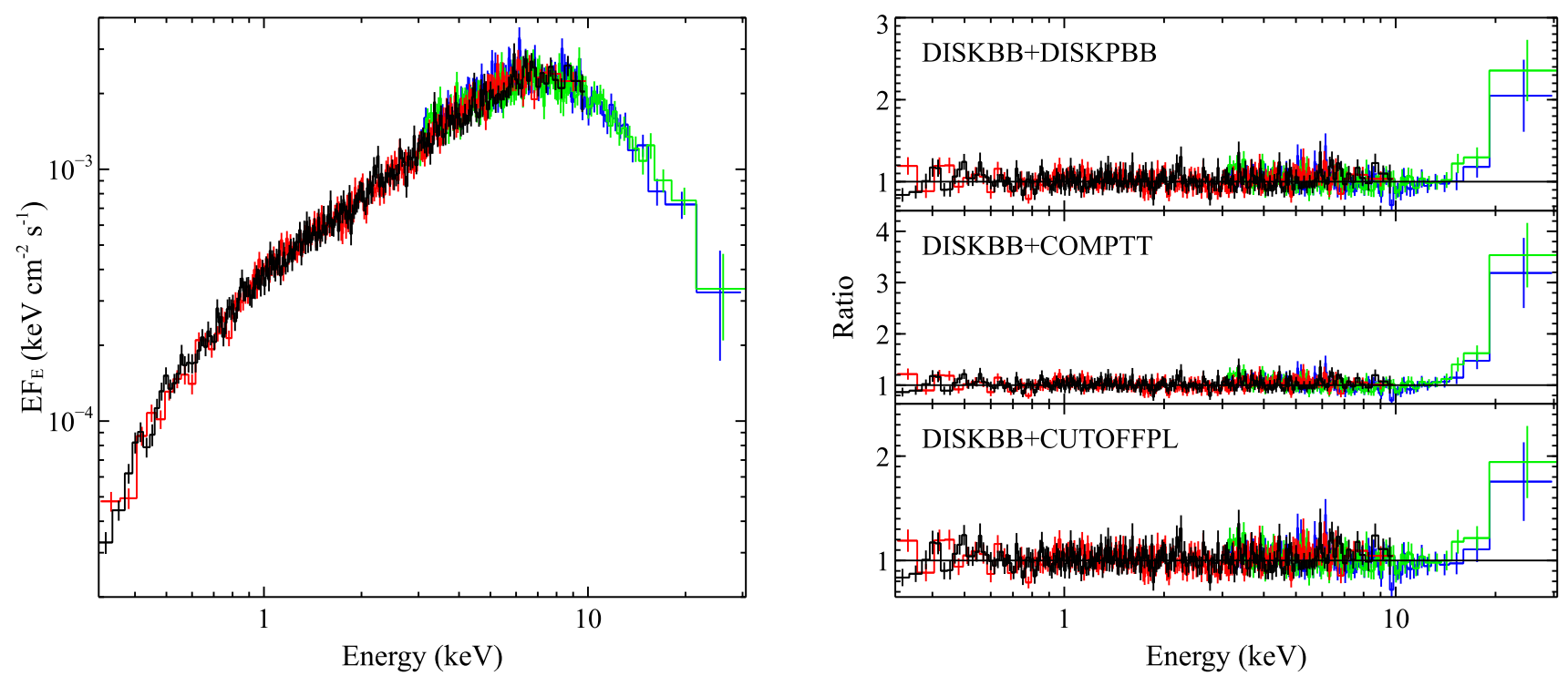

Figure 1. Left-hand panel: the time-averaged broad-band X-ray spectrum of NGC 7793 P13, observed by XMM-Newton (EPIC-pn in black, EPIC-MOS in red) and NUSTAR (FPMA in green, FPMB in blue), unfolded through a model simply consisting of a constant (formally, the model used is a power law with $\Gamma=0$ ). The NUSTAR data clearly demonstrate the presence of a high-energy spectral cutoff. Right-hand panel: data/model ratios for the three continuum models initially considered (see Section 3.1). Each results in a clear excess in the residuals at high energies, indicating the presence of an additional high-energy continuum component. The data have been rebinned for visual clarity.

To further test the presence of this hard excess, we also fit a third model, combining DISKBB with a more phenomenological CUTOFFPL component. This is a simple power-law continuum with an exponential cutoff $\left(E_{\text {cut }}\right)$, which has a broader curvature at high energies than both of the DISKPBB and COMPTT models discussed above. This model has often been applied to ULX data (e.g. Bachetti et al. 2013; Pintore et al. 2017), but is also fairly representative of models typically applied to Galactic neutron stars (e.g. Coburn et al. 2002; Fürst et al. 2014). ${ }^{3}$ However, although it is weaker than both the previous models, even the broader curvature cannot resolve the hard excess (see Fig. 1); a third continuum component is required to fit the time-averaged data regardless of the model used to fit the curvature in the $\sim 5-10 \mathrm{keV}$ band. Similar hard excesses have now been seen in a number of other ULXs observed with NuSTAR (e.g. Mukherjee et al. 2015; Walton et al. 2015b, 2017; Fürst et al. 2017). Following these works, to account for this excess emission we add SIMPL (Steiner et al. 2009) to the higher energy component in the models described above. This is a convolution model that scatters some fraction $\left(f_{\text {scat }}\right)$ of the photons in an input continuum model into a high-energy power-law tail (i.e. photon number is conserved). The additional of SIMPL resolves the hard excess and results in a significant improvement to the fit for all three of the base continuum models considered; even for the DISKBB+CUTOFFPL model - for which the hard excess is weakest - the fit improves by $\Delta \chi^{2}=32$ for two additional free parameters.

In order to confirm that this additional high-energy component is required by the data at a significant level we performed a series of simulations, using the DISKBB+CUTOFFPL continuum model to be conservative, as this is the model that returns the lowest $\Delta \chi^{2}$ when

\footnotetext{
${ }^{3}$ We have also tried replacing the CUTOFFPL model with the FDCUT and NPEX models also frequently applied to Galactic neutron stars (e.g. Odaka et al. 2013; Fürst et al. 2013; Islam et al. 2015; Vybornov et al. 2017), but these provide identical fits to the cutofFPL model, so we do not present the results in full.
}

this component is included in the fit. Using the same response and background files, and adopting the same exposure times as the real data, we simulated 10000 sets of XMM-Newton (pn and combined MOS1+MOS2) and NuSTAR (FPMA and FPMB) spectra with the FAKEIT command in XSPEC based on the best-fitting DISKBB+CUTOFFPL model. Each of the simulated data sets was rebinned in the same manner and analysed over the same bandpass as the real data. We then fit each of the simulated XMM-Newton+NuSTAR data sets with the DISKBB+CUTOFFPL, and noted the $\Delta \chi^{2}$ improvement the addition of a SIMPL component provided over this fit. Of the 10000 data sets simulated, none returned a chance improvement equivalent to or greater than that observed, implying that this component is seen in the real data with a significance comfortably in excess of $3 \sigma$ (for reference, the false-alarm probability for a $3 \sigma$ detection would correspond to $\Delta \chi^{2} \sim 9$ here).

The results for the three models considered here, which all provide statistically equivalent fits, are presented in Table 2 . While the broad-band spectrum is qualitatively similar to the rest of the sample of ULXs observed by NuSTAR to date, there are some notable quantitative differences. For example, the temperature of the lower energy DISKBB component is $k T_{\text {in; DBB }} \sim 0.5 \mathrm{keV}$, slightly hotter than for other ULXs where the same models usually find $k T_{\text {in; DBB }} \sim 0.3 \mathrm{keV}$. Furthermore, the rise of the spectrum up to the peak of the emission at $\sim 7 \mathrm{keV}$ is harder than typically observed from other ULXs. This can be seen in the results for the radial temperature index in the DISKPBB model and the optical depth in the COMPTT model; in the former case we find $p>1.3$ while ULXs more typically show $p<0.75$ when fit with DISKPBB, and in the latter case we find $\tau \sim 17$ while typical ULX values are $\tau \sim 5-10$ with this model. This means the continuum component dominating the $2-10 \mathrm{keV}$ emission is much more peaked (i.e. the spectral curvature in this band is stronger) in P13 than in the broader ULX population. Indeed, if we replace this component with a single-temperature blackbody, a similarly good fit is obtained $\left(\chi^{2} / \mathrm{DoF}=1133 / 1149\right)$, so a range of temperatures is not strongly required in this case. 
Table 2. Best-fitting parameters obtained for the broad-band continuum models applied to the P13 data from epoch XN1.

\begin{tabular}{|c|c|c|c|c|c|c|c|c|c|}
\hline$\overline{\text { Model }^{a}}$ & $\begin{array}{c}N_{\mathrm{H} ; \text { int }} \\
\left(10^{20} \mathrm{~cm}^{-2}\right)\end{array}$ & $\begin{array}{c}k T_{\text {in; DBB }} \\
(\mathrm{keV})\end{array}$ & Norm & $p / \tau / \Gamma_{\mathrm{CPL}}$ & $\begin{array}{c}k T^{b} / E_{\text {cut }} \\
(\mathrm{keV})\end{array}$ & Norm & $\Gamma_{\text {SIMPL }}$ & $\begin{array}{c}f_{\text {scat }} \\
\text { (percent) }\end{array}$ & $\chi^{2} / \mathrm{DoF}$ \\
\hline DISKPBB & $8 \pm 1$ & $0.45_{-0.04}^{+0.03}$ & $1.1_{-0.2}^{+0.4}$ & $>1.3^{c}$ & $2.0 \pm 0.3$ & $8_{-5}^{+6} \times 10^{-2}$ & $3.7_{-1.1}^{+0.9}$ & $>19$ & $1129 / 1148$ \\
\hline СОМРTT & $8 \pm 1$ & $0.49_{-0.20}^{+0.10}$ & $1.0_{-0.5}^{+2.3}$ & $17 \pm 2$ & $1.4_{-0.1}^{+0.3}$ & $7.0_{-1.0}^{+0.6} \times 10^{-4}$ & $4.0_{-0.7}^{+0.3}$ & $>36$ & $1129 / 1148$ \\
\hline CUTOFFPL & $8 \pm 1$ & $0.48_{-0.05}^{+0.08}$ & $1.0 \pm 0.4$ & $-0.9_{-0.6}^{+0.4}$ & $1.9_{-0.6}^{+0.7}$ & $(2.5 \pm 0.5) \times 10^{-4}$ & $4.0_{-1.8}^{+0.7}$ & $>12$ & $1129 / 1148$ \\
\hline
\end{tabular}

Notes. ${ }^{a}$ The base continuum models fit here are e.g. $\mathrm{TBNEW}_{\mathrm{Gal}} \times \mathrm{TBNEW}_{\text {int }} \times(\mathrm{DISKBB}+\mathrm{CUTOFFPL} \otimes \mathrm{SIMPL})$.

${ }^{b}$ Here, $k T$ refers to either the inner temperature for the DISKPBB model, or the electron temperature for the COMPTT model.

${ }^{c}$ During the fitting process, we restrict the radial temperature index to $0.5 \leq p \leq 2.0$.

\subsection{The pulsed emission}

To further characterize the spectral behaviour exhibited by P13, we also investigate spectral variations across the pulse period in an attempt to separate the emission from the accretion column (pulsed) and rest of the accretion flow (which for simplicity is assumed to be steady over the pulse cycle). Owing to its superior time resolution, in this section we are only able to utilize data from the XMM-Newton EPIC-pn detector for the lower energy data; the pulse period is too short to be resolved by the timing capabilities of the EPIC-MOS detectors.

\subsubsection{Difference spectroscopy}

In order to undertake a comparison between P13 and M82 X-2, we compute the spectrum of the pulsed component for the broad-band $X M M-N e w t o n+N u S T A R$ data set. This approach is necessary since the absolute spectrum of M82 X-2 cannot easily be disentangled from the contribution from M $82 \mathrm{X}-1$. We follow the approach taken for M82 X-2 in Brightman et al. (2016a), and subtract the average spectrum obtained during the faintest quarter (i.e. $\Delta \phi_{\text {pulse }}=0.25$ ) of the pulse cycle from that extracted during the brightest quarter (i.e. 'pulse-on'-'pulse-off') in order to isolate the pulsed emission in a simple manner. The data are rebinned to have a minimum $\mathrm{S} / \mathrm{N}$ per energy bin of 3 , to allow the use of $\chi^{2}$ statistics, and we fit the data over the $0.3-20.0 \mathrm{keV}$ energy range with a simple CUTOFFPL model. The intrinsic column density is poorly constrained in these fits, and so we fix it to $8 \times 10^{20} \mathrm{~cm}^{-2}$ following our analysis of the average spectrum, and we also fix the cross-calibration constants between EPIC-pn and FPMA/B to the best-fitting values obtained in that analysis given the low $\mathrm{S} / \mathrm{N}$ of the pulsed spectrum. This provides a good fit to the data, with $\chi^{2}=113$ for 91 DoF. However, for P13 we find the pulsed emission to have a much harder rise, and a lower cutoff energy: $\Gamma=-0.1 \pm 0.3, E_{\text {cut }}=3.9_{-0.6}^{+0.8} \mathrm{keV}$. Identical results are obtained if we minimize the Cash statistic (Cash 1979) instead. For comparison, we show the 2D confidence contours for these parameters in Fig. 2 for both P13 and M82 X-2. The pulsed flux from $\mathrm{P} 13$ during this epoch corresponds to an apparent $0.3-20.0 \mathrm{keV}$ luminosity of $(4.0 \pm 0.3) \times 10^{39} \mathrm{erg} \mathrm{s}^{-1}$ were one to assume isotropic emission (although clearly this is not the case).

\subsubsection{Phase-resolved spectroscopy}

In addition to the 'pulse-on'-'pulse-off' spectroscopy, we also fit the spectra extracted across a series of phase bins. We continue using phase bins of $\Delta \phi_{\text {pulse }}=0.25$ in size, and extract spectra from four periods across the pulse cycle: peak, fall, trough and rise. However, on inspection the fall and rise spectra were found to be practically identical, so we combined them to form a higher $\mathrm{S} / \mathrm{N}$ medium-flux spectrum, resulting in three broad-band spectra to be

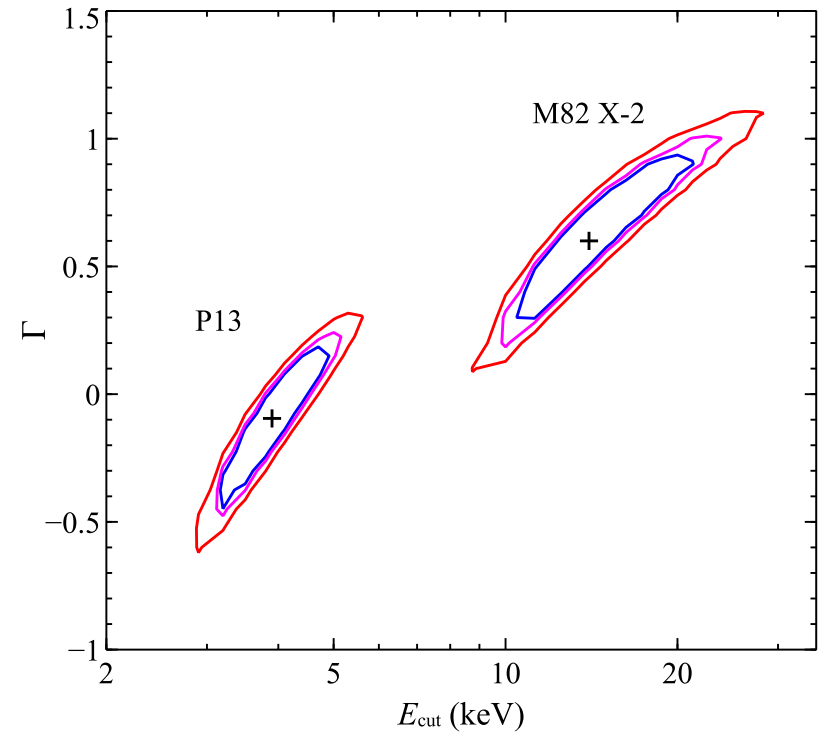

Figure 2. 2D confidence contours for $\Gamma$ and $E_{\text {cut }}$ for the pulsed spectra of P13 and M82 X-2. The 90, 95 and 99 per cent confidence contours for two parameters of interest are shown in blue, magenta and red, respectively.

fit in our phase-resolved analysis: high-flux (peak), medium-flux (rise + fall) and low-flux (trough). We show the high- and low-flux spectra in Fig. 3 for comparison. It is clear that the majority of the difference between the two is seen at higher energies, which is not surprising since the pulse fraction is known to increase with energy (Fürst et al. 2016b; Israel et al. 2017b); the observed spectrum is hardest during the peaks of the pulse cycle.

In order to model these data, we fit all three of the pulse-resolved phase bins simultaneously and undertake a simple decomposition of these spectra into stable and variable (i.e. pulsed) components. Following the previous section, we treat the pulsed emission simply with a CUTOFFPL model. The 'shape' parameters for this component (i.e. $\Gamma, E_{\text {cut }}$ ) are linked across all the phase bins and fixed to the best-fitting results from the difference spectroscopy described above, but its normalization can vary between them. We do not require the normalization of the pulsed CUTOFFPL component to be zero for the low-flux data, as the emission from the accretion column can still contribute during the minimum of the pulse cycle. For the stable emission, we return to the 2-component continuum models discussed previously, and apply the DISKBB+DISKPBB combination. All the parameters for these components, including their normalizations, are linked across all the phase bins, as is the intrinsic neutral absorption column.

The global fit to the phase-resolved data with this approach is excellent $\left(\chi^{2} / \mathrm{DoF}=942 / 932\right)$, and the results are presented in Table 3 . The results for the constant DISKBB+DISKPBB continuum com- 


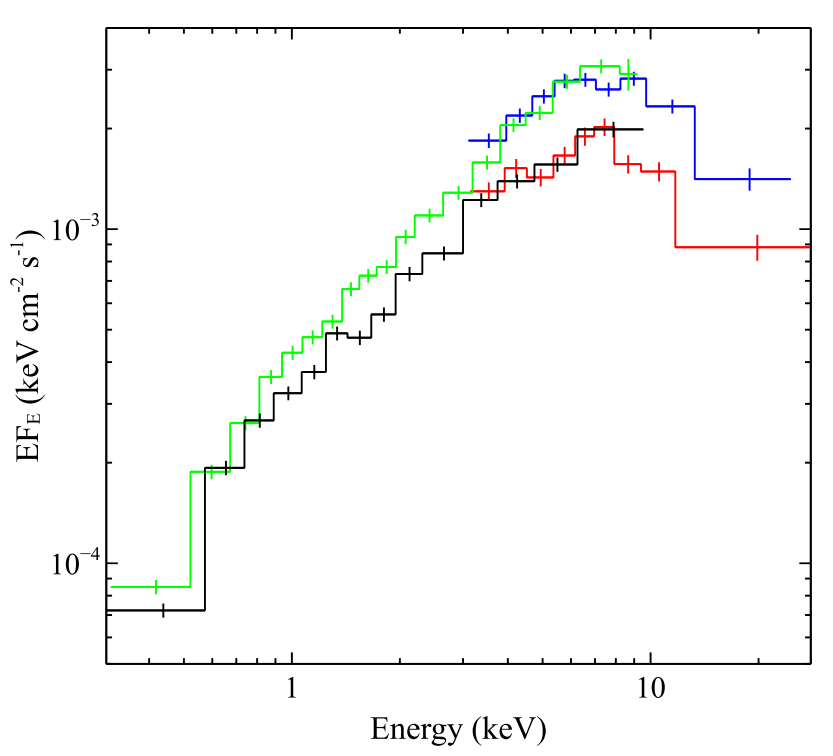

Figure 3. The broad-band X-ray spectrum of NGC 7793 P13 extracted from the peak and the trough of the pulse cycle during epoch XN1. As with Fig. 1 (left-hand panel), the data have been unfolded through a model consisting of a constant. For clarity, the data have been strongly rebinned such that the differences are visually apparent, and we only show the XMM-Newton EPIC-pn (low: black, high: green) and NuSTAR FPMA (low: red, high: blue) data.

Table 3. Best-fitting parameters obtained from our phase-resolved analysis of epoch XN1.

\begin{tabular}{lccc}
\hline Component & \multicolumn{3}{c}{ Parameter } \\
\hline TBABS & $N_{\mathrm{H} ; \text { int }}$ & $\left(10^{20} \mathrm{~cm}^{-2}\right)$ & $8_{-1}^{+2}$ \\
DISKBB & $k T_{\text {in }}$ & $(\mathrm{keV})$ & $0.40_{-0.07}^{+0.01}$ \\
& Norm & & $1.5_{-0.1}^{+1.4}$ \\
DISKPBB & $k T_{\text {in }}$ & $(\mathrm{keV})$ & $1.9 \pm 0.2$ \\
& $p$ & & $>0.95^{a}$ \\
& Norm & $\left(10^{-2}\right)$ & $4.6_{-3.6}^{+0.8}$ \\
CUTOFFPL & $\Gamma$ & & $-0.1^{b}$ \\
& $E_{\text {cut }}$ & $\left(\mathrm{keV}^{c}\right)$ & $3.9^{b}$ \\
& $F_{2-10^{c}}$ (low) & $\left(10^{-12} \mathrm{erg} \mathrm{cm}^{-2} \mathrm{~s}^{-1}\right)$ & $1.7_{-0.3}^{+0.2}$ \\
& $F_{2-10^{c}}$ (medium) & $\left(10^{-12} \mathrm{erg} \mathrm{cm}^{-2} \mathrm{~s}^{-1}\right)$ & $2.6_{-0.3}^{+0.2}$ \\
& $F_{2-10^{c}}$ (high) & $\left(10^{-12} \mathrm{erg} \mathrm{cm}^{-2} \mathrm{~s}^{-1}\right)$ & $3.6_{-0.3}^{+0.2}$ \\
$\chi^{2} /$ DoF & & & $942 / 932$ \\
\hline
\end{tabular}

Notes. ${ }^{a}$ As before, we restrict the radial temperature index to $0.5 \leq p \leq 2.0$.

${ }^{b}$ These parameters have been fixed to the best-fitting values from the 'pulseon'-'pulse-off' difference spectroscopy (Section 3.2.1).

${ }^{c}$ Observed fluxes for the CUTOFFPL component in the $2-10 \mathrm{keV}$ band.

ponents are broadly similar to the results obtained with the model utilizing this combination in our time-averaged spectral analysis (see Table 2). This suggests that for the case of P13, the need for the SIMPL component in the time-averaged models is largely driven by the contribution of the pulsed emission. The relative contribution of the DISKBB, DISKPBB and CUTOFFPL components in our analysis is shown for the peaks and the troughs of the pulse cycle in Fig. 4; the CUTOFFPL model dominates the flux at the highest energies probed by $N U S T A R$, exactly where the SIMPL component contributes in the time-averaged models. Indeed, the steady continuum emission does not require an additional high-energy power-law tail in

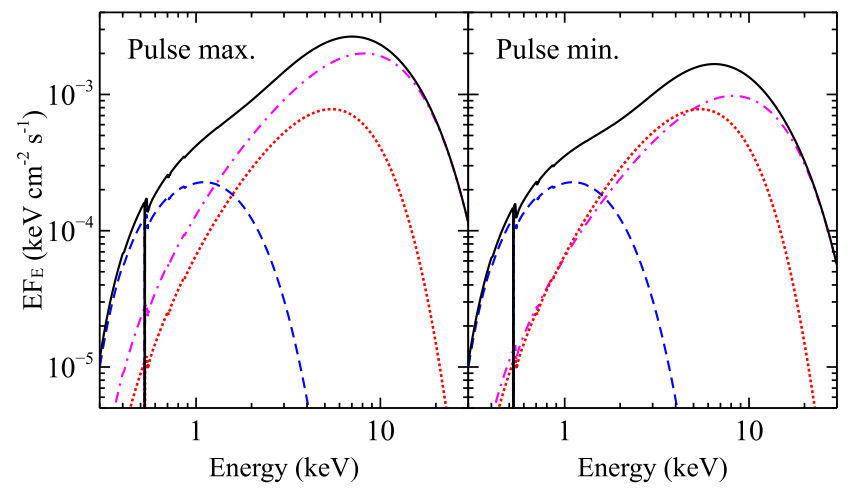

Figure 4. The relative contributions of the various spectral components during the peaks (left-hand panel) and the troughs (right-hand panel) of the pulse cycle from our phase-resolved analysis of epoch XN1. In both panels the total model is shown in solid black, the DISKBB component (steady) in dashed blue, the DISKPBB component (steady) in dotted red and the CUTOFFPL component (pulsed) in dash-dotted magenta. The DISKBB and DISKPBB components are assumed to be steady across the pulse cycle, and are identical in both panels.

these fits; adding a SIMPL component to the DISKPBB component only improves the fit by $\Delta \chi^{2}=6$ for two additional free parameters (although the situations are not identical, this would be below the threshold required to claim a significant detection based on the simulations presented above). We stress though that both the DISKBB and DISKPBB components are required to describe the phase-resolved data; removing either significantly degrades the global fit. Three continuum components are therefore required to fully describe the phase-resolved data from epoch XN1: one pulsed component and two steady components.

\section{NGC 7793 P13: MULTI-EPOCH ANALYSIS}

We next undertake a multi-epoch analysis for P13 additionally including the archival XMM-Newton data sets (see Table 1). A comparison of the average spectra from each of these observations with the broad-band $X M M-N e w t o n+N u S T A R$ data set is shown in Fig. 5. The first of the archival XMM-Newton observations (epoch X1) was taken during the extended off-state that spanned mid-2011 to mid-2013 (Motch et al. 2014; Fürst et al. 2016b) and subsequently only a very low $\mathrm{S} / \mathrm{N}$ spectrum is available. However, the latter two both caught P13 at ULX luminosities, with fluxes only a factor of $\sim 4$ and $\sim 2$ fainter than epoch XN1 during epochs X2 and X3, respectively (see Table 4). For the ULX-luminosity observations, the $0.3-10.0 \mathrm{keV}$ spectrum of P13 is clearly harder when the source is brighter, and exhibits stronger variations at the higher energies of this bandpass. During the faintest of these observations (epoch $\mathrm{X} 2$ ) the spectrum is most visibly doubly peaked, as the relative contribution of the soft thermal component is strongest during this epoch.

\subsection{Evolution of the pulsed emission}

As noted by Fürst et al. (2016b), during epoch X1 P13 was too faint for any pulsations to be detectable. In addition, none of the Chandra observations have sufficient temporal resolution to see the pulsations. However, pulsations were detected in all the subsequent epochs (X2, X3 and XN1). Table 4 summarizes the results obtained from the pulsation searches presented in Fürst et al. (2016b), showing a clear spin-up. In addition to these results, we also compute 
Table 4. Evolution in the properties of the X-ray pulsations across the various epochs considered.

\begin{tabular}{lccccc}
\hline Epoch & $\begin{array}{c}F_{\mathrm{av}, 0.3-10} \\
\left(10^{-12} \mathrm{erg} \mathrm{cm}^{-2} \mathrm{~s}^{-1}\right)\end{array}$ & $\begin{array}{c}P \\
(\mathrm{~ms})\end{array}$ & $\begin{array}{c}P_{\text {frac }, 0.5-2.0} \\
(\text { per cent })\end{array}$ & $\begin{array}{c}P_{\text {frac, } 2-10} \\
(\text { per cent })\end{array}$ & $\begin{array}{c}F_{\text {pulse, } 2-10} \\
\left(10^{-12} \mathrm{erg} \mathrm{cm}^{-2} \mathrm{~s}^{-1}\right)\end{array}$ \\
\hline $\mathrm{X} 2$ & $1.14 \pm 0.03$ & $419.712 \pm 0.008$ & $12 \pm 2$ & $31 \pm 3$ & $0.57 \pm 0.08$ \\
$\mathrm{X} 3$ & $2.84 \pm 0.05$ & $418.390 \pm 0.008$ & $18 \pm 2$ & $29 \pm 2$ & $1.19 \pm 0.12$ \\
$\mathrm{XN1}$ & $5.19 \pm 0.07$ & $416.9513 \pm 0.0017$ & $13 \pm 1$ & $22 \pm 1$ & $1.84 \pm 0.12$ \\
\hline
\end{tabular}

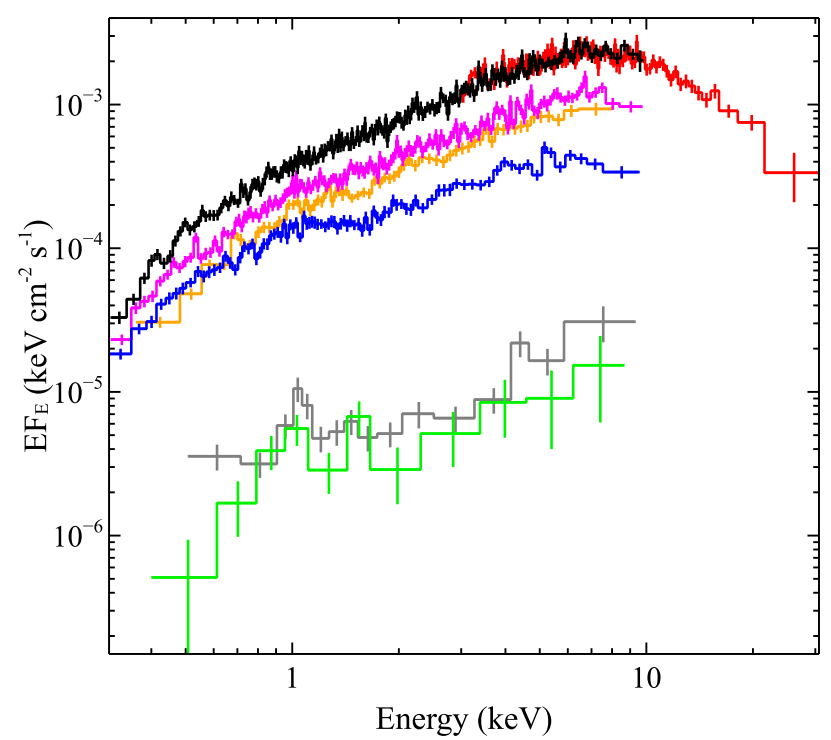

Figure 5. A comparison of the time-averaged spectra of P13 from each of the epochs considered in this work, again unfolded through a simple model that is constant with energy. For clarity, we only show the XMM-Newton EPIC-pn and NUSTAR FPMA data. The data from epochs XN1, X1, X2, X3, $\mathrm{C} 1$ and $\mathrm{C} 2+\mathrm{C} 3+\mathrm{C} 4$ (see Section 4.3) are shown in black (EPIC-pn) and red (FPMA), green, blue, magenta, orange and grey, respectively. As before, the data have been rebinned for visual purposes.

the pulse fractions for the $0.5-2.0$ and $2.0-10.0 \mathrm{keV}$ bands that are common to all three epochs where pulsations have been detected, based on the XMM-Newton EPIC-pn light curves. Here, we calculate the pulse fraction as $(\max -\min ) /(\max +\min )$, where $\max$ and min refer to the average count rates at the maximum and minimum phases of the pulse cycle. The results are shown in Fig. 6.

As expected, the pulse fractions are systematically higher in the harder $2.0-10.0 \mathrm{keV}$ band. At these energies, the pulse fraction shows evidence for a secular decrease with increasing observed flux. This suggests that there are two emission components contributing in the $2.0-10.0 \mathrm{keV}$ band (pulsed and non-pulsed), and that the relative contribution from the pulsed component is lower at higher fluxes. In contrast, in the softer band the pulse fraction initially increases with flux, and then decreases again at even higher fluxes. The evolution seen at these energies between epochs X3 and $\mathrm{XN1}$ (the two higher flux observations) is likely related to the same effect producing the anticorrelation in the harder band, i.e. the relative contribution of the pulsed component decreasing relative to some non-pulsed component as the flux increases. The switch to a decreasing pulse fraction with decreasing flux is then likely related to the increased relative importance of the cooler thermal component during epoch X2, which is visually apparent from Fig. 5. This implies that the cooler thermal component is also not pulsed, resulting in the lower soft-band pulse fractions.

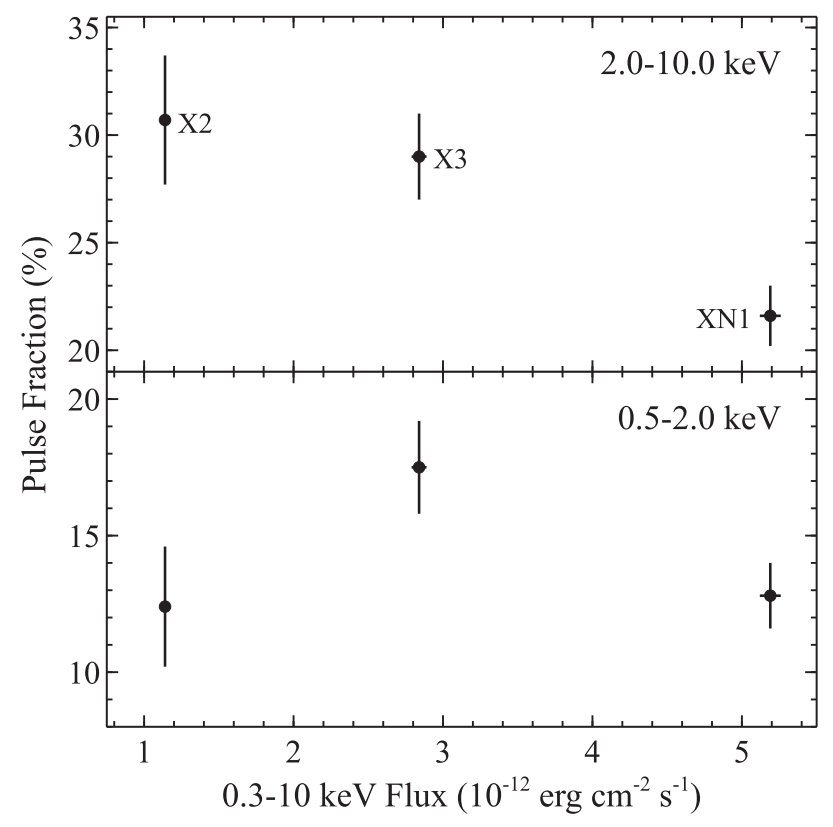

Figure 6. Evolution of the pulse fraction in two energy bands ( $0.5-2.0$ and $2.0-10.0 \mathrm{keV}$ ) as a function of observed $0.3-10.0 \mathrm{keV}$ flux.

The relative evolutions seen in the pulse fractions for these two bands therefore suggests that there are two emission components that contribute to both the hard and the soft bands (pulsed and nonpulsed), and a third non-pulsed component that only contributes to the soft band. This is broadly similar to the spectral decomposition presented in Section 3.2.

We also investigate the spectral evolution of the pulsed emission, by repeating the 'pulse-on'-'pulse-off' analysis presented in Section 3.2.1 for the two relevant archival XMM-Newton observations. As before, here we can only make use of the EPIC-pn data from $X M M-$ Newton for epochs $\mathrm{X} 2$ and $\mathrm{X} 3$. We fit the pulsed emission from all three epochs (X2, X3 and XN1) simultaneously, using the CUTOFFPL model again. Owing to the limited bandpass available for epochs X2 and X3, we link the high-energy cutoff between all epochs so that we can undertake a self-consistent comparison of the slope of the pulsed emission, given the degeneracy between $\Gamma$ and $E_{\text {cut }}$ that can be seen in Fig. 2. In Fig. 7, we show the 2D confidence contours for the photon index and the $2-10 \mathrm{keV}$ flux for the pulsed emission from all three epochs. Although the pulsed flux does vary, in all three cases the spectral forms are clearly consistent. We therefore perform a final fit to the pulsed spectra in which $\Gamma$ is linked across all the data sets as well, leaving only the normalization of the CUTOFFPL model free to vary between them. With this approach, we find $\Gamma=0.17_{-0.19}^{+0.17}$ and $E_{\text {cut }}=4.7_{-0.7}^{+0.9} \mathrm{keV}$, consistent with the results obtained fitting the pulsed data from epoch XN1 only. The pulsed fluxes computed with this final analysis are given in Table 4. 


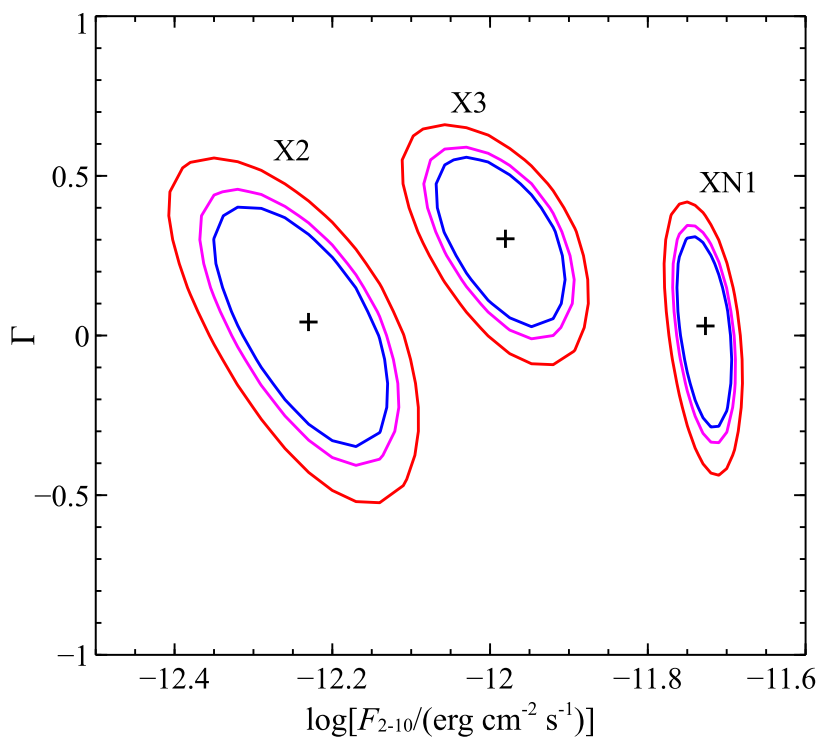

Figure 7. $2 \mathrm{D}$ confidence contours for $\Gamma$ and the $2-10 \mathrm{keV}$ flux for the pulsed spectra of P13 from epochs X2, X3 and XN1. As in Fig. 2, the 90, 95 and 99 percent confidence contours for two parameters of interest are shown in blue, magenta and red, respectively.

\subsection{Time-averaged analysis of the ULX state}

We now undertake a simultaneous, multi-epoch analysis of the average spectra extracted from the epochs during which P13 exhibited ULX luminosities. We take a similar approach to our analysis of the phase-resolved spectra from epoch XN1 (see Section 3.2.2) and fit the data from each epoch with three continuum components: a CUTOFFPL model to account for the pulsed emission, and an underlying 2-component continuum to account for the non-pulsed emission, again utilizing the DISKBB+DISKPBB combination. Having found that the spectral shape of the pulsed emission remains constant throughout all the epochs from which pulsations could be detected to date, we utilize this in our multi-epoch analysis and again fix the shape parameters $\left(\Gamma, E_{\text {cut }}\right)$ of the CUTOFFPL model to the best-fitting values obtained from our analysis of the pulsed spectra, taking the values from our global fit to the pulsed emission from epochs X2, X3 and XN1 (see Section 4.1).

Given this approach, we focus here on epochs X2, X3 and XN1, since we are not able to resolve the pulsations in the Chandra data from epoch $\mathrm{C} 1$. However, we do note that the average spectrum from epoch $\mathrm{C} 1$ is very similar to epoch $\mathrm{X} 3$ (see Fig. 5), so similar results would be expected. We fit the data from all three epochs simultaneously in order to investigate which of the spectral parameters drive the observed variability. This also allows us to make the simplifying assumption that the total flux from the accretion column is proportional to the pulsed flux. As such, we assume that the average CUTOFFPL flux in the $2-10 \mathrm{keV}$ band (common to all epochs) shows the same relative variations as the pulsed flux from epoch-to-epoch (see Fig. 7 and Table 4); the fluxes of the pulsed emission across all three epochs are therefore controlled by a single free parameter in our analysis, which we choose to be the pulsed flux in epoch XN1.

We also assume that the intrinsic neutral absorption column does not vary between the epochs considered (see e.g. Miller et al. 2013). Furthermore, during our analysis we find that the radial temperature index for the DISKPBB component $(p)$ can be linked across all epochs; doing so only worsens the fit by $\Delta \chi^{2}=1$ for two fewer free parameters (an $F$-test ${ }^{4}$ confirms this improvement is not significant, with a false-alarm probability of 0.6). Both of the DISKBB and DISKPBB temperatures are found to vary. Linking either of these across all three epochs degrades the fit by $\Delta \chi^{2}>22$ (again for two fewer free parameters; here the $F$-test gives a false-alarm probability of $<3 \times 10^{-5}$ ). The global fit for the model in which $N_{\mathrm{H} \text {, int }}$ and $p$ are linked across all epochs is very good, with $\chi^{2} / \mathrm{DoF}=2149 / 2058$, and the parameter constraints are presented in Table 5 .

One notable result common to all our spectral analyses is that the radial temperature index for the DISKPBB component is rather steep; here we find $p>1.52$ (see also Sections 3.1 and 3.2.2). This implies that the emission from this component is significantly more peaked, i.e. dominated by a narrow range of temperatures, than a standard thin disc continuum (which should give $p=0.75$; Shakura \& Sunyaev 1973). We therefore test here whether a range of temperatures is really required for this component, and replace DISKPBB with a single blackbody (вв) component. The fit in this case is actually slightly improved over the DISKPBB model, despite having one fewer free parameter: $\chi^{2} / \mathrm{DoF}=2144 / 2059$ (this is likely due to the upper limit of 2.0 we place on $p$ in our previous models). In contrast, the lower temperature component does require a range of temperatures; replacing the DISKBB component with a single вв worsens the fit by $\Delta \chi^{2}>30$ (for the same number of free parameters) regardless of the model used for the hotter component (DISKPBB or BB).

The results for the model in which the hotter component is treated as a single вв are also given in Table 5, and we show the data/model ratios for each epoch in Fig. 8. We also show the relative contributions of the various components for the best-fitting model in Fig. 9; the evolution of the various components is qualitatively consistent with that expected from the evolution in the soft- and hard-band pulse fractions (Fig. 6). Lastly, as a further sanity check, we compare the total flux for the CUTOFFPL model in epoch XN1 (against which the other epochs are scaled) obtained with this model to that expected based on the phase-resolved analysis of this epoch (Section 3.2.2) and the pulsed flux observed (Table 4). The flux for the CUTOFFPL component obtained for the minimum of the pulse cycle is $\sim 1.7 \times 10^{-12} \mathrm{erg} \mathrm{cm}^{-2} \mathrm{~s}^{-1}$ in the $2-10 \mathrm{keV}$ band (see Table 3 ). For a perfectly symmetric sinusoidal pulse profile, the total CUTOFFPL flux would be expected to be the sum of this flux and half of the pulsed flux, i.e. $\sim 2.6 \times 10^{-12} \mathrm{erg} \mathrm{cm}^{-2} \mathrm{~s}^{-1}$. This is very similar to the total CUTOFFPL flux obtained for epoch XN1 in our multi-epoch spectral analysis with the DISKBB+BB+CUTOFFPL model (Table 5), particularly when accounting for statistical uncertainties and the fact that the pulse profile is not perfectly sinusoidal (Fürst et al. 2016b), suggesting that our spectral decomposition in this case is internally self-consistent.

\subsection{The low-flux observations}

In addition to the ULX luminosity observations, we also undertake a simple analysis of the average spectra extracted from the observations taken during the off-state (epochs C2, C3, C4 and X1). Although there are some flux variations, the spectra from these epochs are all broadly consistent (key spectral parameters agree within their 90 percent errors when fit individually), so to maximize the $\mathrm{S} / \mathrm{N}$ we co-add the Chandra data to form a single spectrum using ADDASCASPEC. The combined Chandra spectrum from epochs $\mathrm{C} 2+\mathrm{C} 3+\mathrm{C} 4$ and the EPIC-pn data from epoch $\mathrm{X} 1$

\footnotetext{
${ }^{4}$ See Protassov et al. (2002) for appropriate uses of the $F$-test.
} 
Table 5. Results obtained for the multi-epoch analysis of P13.

\begin{tabular}{|c|c|c|c|c|c|c|}
\hline \multirow{2}{*}{$\begin{array}{l}\text { Model } \\
\text { component }\end{array}$} & \multicolumn{2}{|r|}{ Parameter } & \multirow[t]{2}{*}{ Global } & \multicolumn{3}{|c|}{ Epoch } \\
\hline & & & & $\mathrm{X} 2$ & $\mathrm{X} 3$ & $\mathrm{XN1}$ \\
\hline \multicolumn{7}{|c|}{ Continuum: DISKBB+DISKPBB+CUTOFFPL } \\
\hline TBABS & $N_{\mathrm{H}, \text { int }}$ & $\left(10^{20} \mathrm{~cm}^{-2}\right)$ & $7.7 \pm 0.8$ & & & \\
\hline \multirow[t]{2}{*}{ DISKBB } & $T_{\text {in }}$ & $(\mathrm{keV})$ & & $0.34 \pm 0.01$ & $0.417_{-0.026}^{+0.008}$ & $0.421_{-0.022}^{+0.009}$ \\
\hline & Norm & & & $1.24_{-0.10}^{+0.32}$ & $0.85_{-0.07}^{+0.25}$ & $1.25_{-0.11}^{+0.25}$ \\
\hline \multirow[t]{3}{*}{ DISKPBB } & $T_{\text {in }}$ & $(\mathrm{keV})$ & & $1.70 \pm 0.15$ & $2.14_{-0.11}^{+0.14}$ & $2.15_{-0.06}^{+0.10}$ \\
\hline & $p$ & & $>1.52^{a}$ & & & \\
\hline & Norm & $\left(10^{-2}\right)$ & & $1.3_{-0.5}^{+0.4}$ & $1.9_{-0.7}^{+0.3}$ & $4.0_{-1.4}^{+0.1}$ \\
\hline \multirow[t]{3}{*}{ CUTOFFPL } & $\Gamma$ & & $0.17^{b}$ & & & \\
\hline & $E_{\text {cut }}$ & $(\mathrm{keV})$ & $4.7^{b}$ & & & \\
\hline & $F_{2-10}$ & $\left(10^{-13} \mathrm{erg} \mathrm{cm}^{-2} \mathrm{~s}^{-1}\right)$ & & $5.5^{c}$ & $11.3^{c}$ & $17.6_{-2.8}^{+2.0}$ \\
\hline \multirow[t]{2}{*}{$\chi^{2} / \mathrm{DoF}$} & & & $2149 / 2058$ & & & \\
\hline & & Continuum: D & $\mathrm{KBB}+\mathrm{BB}+$ & JTOFFPL & & \\
\hline TBABS & $N_{\mathrm{H}, \text { int }}$ & $\left(10^{20} \mathrm{~cm}^{-2}\right)$ & $7.1_{-0.7}^{+0.8}$ & & & \\
\hline \multirow[t]{2}{*}{ DISKBB } & $T_{\text {in }}$ & $(\mathrm{keV})$ & & $0.35 \pm 0.02$ & $0.45 \pm 0.03$ & $0.48 \pm 0.03$ \\
\hline & Norm & & & $1.05_{-0.21}^{+0.27}$ & $0.63_{-0.13}^{+0.17}$ & $0.80_{-0.15}^{+0.19}$ \\
\hline \multirow[t]{2}{*}{ вв } & $T_{\text {in }}$ & $(\mathrm{keV})$ & & $1.1 \pm 0.1$ & $1.5 \pm 0.1$ & $1.53 \pm 0.04$ \\
\hline & Norm & $\left(10^{-6}\right)$ & & $2.4 \pm 0.6$ & $11.1 \pm 1.5$ & $27.3_{-2.2}^{+2.4}$ \\
\hline \multirow[t]{3}{*}{ CUTOFFPL } & $\Gamma$ & & $0.17^{b}$ & & & \\
\hline & $E_{\text {cut }}$ & $(\mathrm{keV})$ & $4.7^{b}$ & & & \\
\hline & $F_{2-10}$ & $\left(10^{-13} \mathrm{erg} \mathrm{cm}^{-2} \mathrm{~s}^{-1}\right)$ & & $6.9^{c}$ & $14.4^{c}$ & $22.3_{-1.6}^{+1.4}$ \\
\hline$\chi^{2} / \mathrm{DoF}$ & & & $2144 / 2059$ & & & \\
\hline
\end{tabular}

Notes. ${ }^{a}$ As before, we restrict the radial temperature index to $0.5 \leq p \leq 2.0$.

${ }^{b}$ These parameters have been fixed to the best-fitting values from the global fit to the multi-epoch 'pulse-on''pulse-off' difference spectra (Section 4.1).

${ }^{c}$ The CUTOFFPL fluxes for epochs X2 and X3 are scaled relative to epoch XN1 based on the relative pulsed fluxes seen from these epochs (see Table 4).

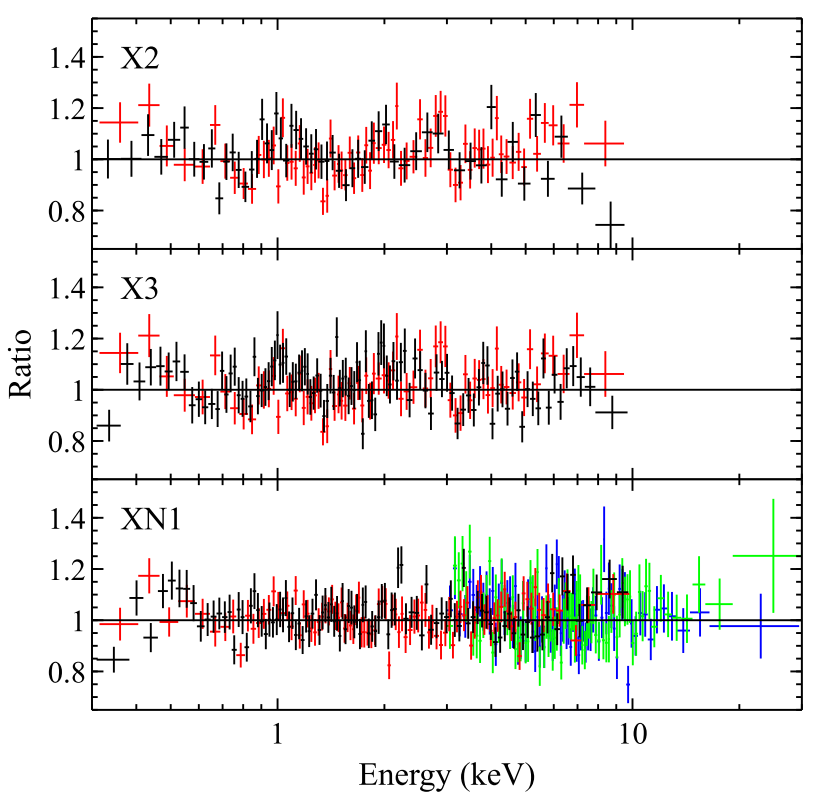

Figure 8. Data/model ratios for our multi-epoch analysis of the time-averaged spectra from epochs X2 (top), X3 (middle) and XN1 (bottom; see Section 4.2). The ratios are plotted for the model combining DISKBB+BB+CUTOFFPL for the intrinsic continuum; the colour-coding of the different data sets is the same as Fig. 1. are shown in Fig. 5. In order to get the best constraints for this state, we fit these Chandra and XMM-Newton data sets simultaneously with some simple models. We first apply a simple absorbed power-law continuum, fixing the intrinsic neutral absorption to $N_{\mathrm{H} \text {; int }}=8 \times 10^{20} \mathrm{~cm}^{-2}$ based on the fits to the broad-band data, and assuming a common photon index for the Chandra and XMMNewton data sets. However, we find that this does not fit the data $\left(\chi^{2} / \mathrm{DoF}=63 / 29\right)$ and leaves obvious residuals. These are particularly prominent around $\sim 1 \mathrm{keV}$ (see Fig. 10), and are reminiscent of the contribution from thermal plasma emission at low energies.

We therefore add a MEKAL plasma component to the model. We assume this is only absorbed by Galactic absorption, that this component has solar abundances, and that the temperature remains constant between the Chandra and XMM-Newton data sets, but we also find that the normalizations for this component are consistent between the data sets, and therefore also link this parameter in our final model. The only parameter that is allowed to vary between the XMM-Newton and Chandra spectra is the normalization of the power-law component. This model fits the off-state data very well $\left(\chi^{2} / \mathrm{DoF}=28 / 27\right)$, and resolves the $\sim 1 \mathrm{keV}$ residuals. We stress that both the MEKAL and the power-law components are required to fit the data; removing the latter results in a significantly worse fit again $\left(\chi^{2} / \mathrm{DoF}=61 / 29\right)$. The best-fitting model for the Chandra spectrum is shown in Fig. 10, and the parameter constraints are given in Table 6.

The MEKAL component contributes at low energies, fitting the $\sim 1 \mathrm{keV}$ feature with iron L emission, but in addition to this a very 


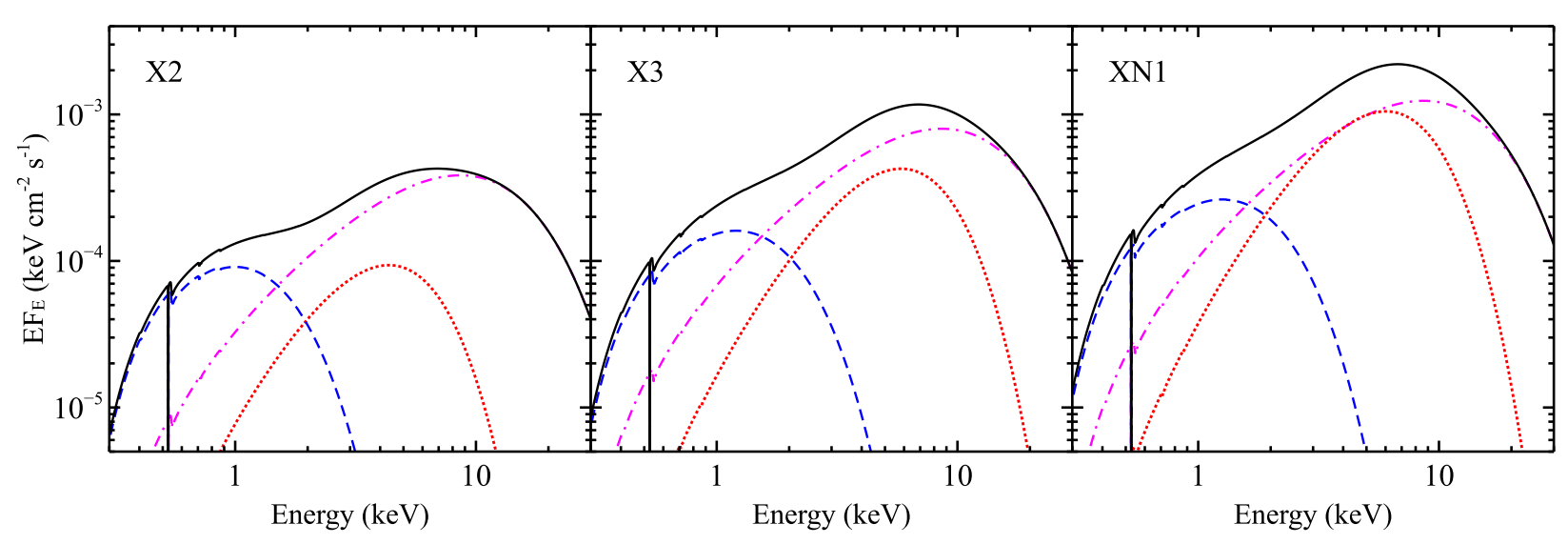

Figure 9. The relative contributions of the various spectral components from our multi-epoch analysis of the time-averaged spectra from epochs X2 (left), X3 (middle) and XN1 (right). In all panels the total model is shown in solid black, the DISKBB component (steady) in dashed blue, the BB component (steady) in dotted red and the CUTOFFPL component (pulsed) in dash-dotted magenta, respectively.

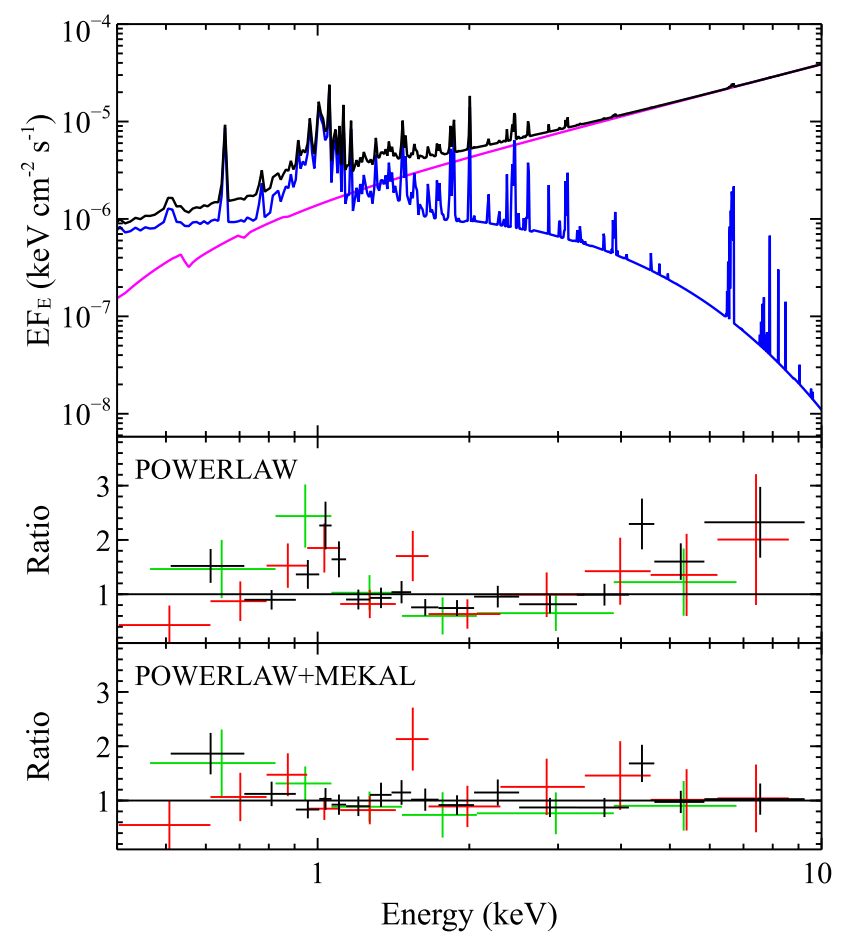

Figure 10. Top panel:the best-fitting model for the integrated Chandra data obtained during the off-state (i.e. epochs $\mathrm{C} 2+\mathrm{C} 3+\mathrm{C} 4$; see Section 4.3). The total model, and the relative contributions of the power-law and the MEKAL components are shown in black, magenta and blue, respectively. Middle panel: data/model ratio for the power-law-only model, fit to both the Chandra and XMM-Newton off-state data sets. Clear residuals remain, particularly at $\sim 1 \mathrm{keV}$. Bottom panel: same as the middle panel, but for the power-law + MEKAL model. In both the middle and bottom panels, the Chandra ACIS data and the XMM-Newton EPIC-pn and EPIC-MOS data are shown in black, red and green, respectively.

hard power-law continuum $\left(\Gamma=0.7_{-0.5}^{+0.4}\right)$ is required at higher energies, which we assume to be related to residual accretion on to the neutron star. However, the fact that the normalizations for the MEKAL component are consistent for the XMM-Newton and Chan$d r a$ data sets, despite their significantly different extraction regions, suggests that this is not faint, extended emission from the broader galaxy, but is likely also be associated with P13. However, even if
Table 6. Best-fitting parameters obtained for the off-state data sets.

\begin{tabular}{lcccc}
\hline Parameter & & Global & Chandra & $X M M$ \\
\hline$k T_{\text {MEKAL }}$ & $(\mathrm{keV})$ & $1.3_{-0.2}^{+0.3}$ & & \\
Norm & {$\left[\left(10^{-6}\right)\right.$} & $5 \pm 2$ & & \\
$\Gamma$ & & $0.7_{-0.5}^{+0.4}$ & & \\
Norm & $\left(10^{-6}\right)$ & & $1.8_{-0.9}^{+1.2}$ & $0.8_{-0.5}^{+0.9}$ \\
$\chi^{2} / \mathrm{DoF}$ & & $28 / 27$ & & \\
$L_{\mathrm{tot}}{ }^{a}$ & $\left(10^{37} \mathrm{erg} \mathrm{s}^{-1}\right)$ & & $7.9_{-1.3}^{+1.2}$ & $4.5_{-1.6}^{+1.4}$ \\
$L_{\mathrm{po}}{ }^{a}$ & & & $6.5 \pm 1.4$ & $3.0_{-1.7}^{+1.2}$ \\
\hline
\end{tabular}

Note. ${ }^{a}$ Observed luminosities in the $0.3-10.0 \mathrm{keV}$ band for the total emission and the power-law component, respectively, assuming isotropic emission.

this is the case it is not clear that this emission is directly powered by accretion, so we compute the observed fluxes for both the total emission and just the power-law component (see Table 6). The latter provides the majority of the observed flux in the $0.3-10.0 \mathrm{keV}$ band in both the Chandra and the XMM-Newton data sets; the residual accretion power is orders of magnitude lower than the peak flux observed from epoch XN1, regardless of the power source for the plasma emission.

\section{THE BROAD-BAND ULX SAMPLE}

For comparison with NGC7793 P13, we also compiled the sample of ULXs with broad-band spectra available in the literature at the time of writing. We focus only on sources for which NUSTAR has provided a robust detection out at least $20 \mathrm{keV}$, which are not significantly confused with any other X-ray sources, and for which simultaneous data from at least one other mission with high S/N soft X-ray coverage (XMM-Newton and/or Suzaku) exist. This sample includes: Circinus ULX5 (Walton et al. 2013), Holmberg II X-1 (Walton et al. 2015b), Holmberg IX X-1 (Walton et al. 2014, 2017; Luangtip, Roberts \& Done 2016), IC 342 X-1 and X-2 (Rana et al. 2015), NGC 1313 X-1 (Bachetti et al. 2013; Miller et al. 2014; Walton et al. 2016a), NGC 5204 X-1 (Mukherjee et al. 2015) and NGC 5907 ULX (Walton et al. 2015a; Fürst et al. 2017; Israel et al. 2017a). Where necessary, we reprocessed the data with the latest calibrations, largely following the reduction procedures outlined in the referenced works, and also included the NUSTAR 'spacecraft science' data (see Section 2). Notable exclu- 
sions are NGC $1313 \mathrm{X}-2$, which is not detected much above $10 \mathrm{keV}$ (Bachetti et al. 2013), and M82 X-1 and X-2, which are strongly blended in the NuSTAR data (e.g. Bachetti et al. 2014; Brightman et al. 2016a,b). We also do not consider NGC 5643 X-1 or M51 X-8; both are only weakly detected by NUSTAR above $10 \mathrm{keV}$ in short observations, and neither of the NUSTAR observations of these sources have simultaneous high $\mathrm{S} / \mathrm{N}$ soft X-ray coverage (Annuar et al. 2015; Earnshaw et al. 2016; Krivonos \& Sazonov 2016). Finally, we also note that there is a further NuSTAR observation of IC 342 that we also do not include, as again this has no simultaneous high S/N soft X-ray coverage (Shidatsu, Ueda \& Fabrika 2017). The majority of the sources considered here are also included in the ULX sample recently presented by Pintore et al. (2017).

The time-averaged broad-band spectra for the selected sample, including NGC 7793 P13, are shown in Fig. 11 (in the case of Holmberg IX X-1, we only show three of the six broad-band observations available, corresponding to epochs 1,2 and 5 as defined in Walton et al. 2017; the remaining three are similar to either the low or medium-flux states shown). There is clearly some diversity among the observed spectra; the lowest energies in particular are influenced by different levels of neutral absorption. However, there are also many similarities. For all the sources with low absorption, evidence for two thermal continuum components can be seen in the spectra below $10 \mathrm{keV}$ (although the relative contributions of these components can vary both between sources and over time for an individual source, also helping to increase the diversity of the observed spectral shapes), similar to P13. Motch et al. (2014) also note that the spectrum of $\mathrm{P} 13$ below $10 \mathrm{keV}$ is fairly representative of the broader ULX population. The qualitative similarity of the NuSTAR data in all cases is particularly striking; all the high-energy spectra break in a fairly narrow energy band above $\sim 3 \mathrm{keV}$ to a steep continuum above $10 \mathrm{keV}$. Evidence for an additional high-energy power-law tail (or at least, an additional high-energy continuum component) has now been seen in several other systems in addition to P13 (Circinus ULX5: Walton et al. 2013; Holmberg II X-1: Walton et al. 2015b; Holmberg IX X-1: Walton et al. 2014, 2017; NGC 5204 X-1: Mukherjee et al. 2015; NGC 5907 ULX: Fürst et al. 2017). Despite the clear diversity among the spectra, the basic spectral components present in the broader ULX population, for which the nature of the accretors generally remains unknown, therefore appear similar to NGC 7793 P13 (see also Pintore et al. 2017).

\section{DISCUSSION}

We have performed a detailed spectral analysis of the ULX NGC 7793 P13, focusing on the first high-energy detection of the source by NUSTAR. The recent detection of X-ray pulsations (Fürst et al. 2016b; Israel et al. 2017b) firmly identify it as a neutron star accretor, meaning the peak luminosity observed $\left(L_{\mathrm{X} \text {, peak }} \sim 10^{40} \mathrm{erg} \mathrm{s}^{-1}\right)$ is highly super-Eddington. The low level of absorption towards P13 and the lack of source confusion makes it a key laboratory for understanding the super-Eddington accretion on to neutron stars, and also their relation to the broader ULX population. Through a combination of time-averaged, phase-resolved and multi-epoch analyses, we find that three spectral components are required to fit the data: two thermal blackbody components that contribute below $\sim 10 \mathrm{keV}$ (with temperatures of the hotter and cooler components varying from $\sim 1$ to $1.5 \mathrm{keV}$ and from $\sim 0.3$ to $0.5 \mathrm{keV}$ between the observed epochs, respectively), and a third component that extends to higher energies. As discussed in Section 5 , these components are qualitatively similar to those seen in other ULXs with broad-band coverage.

\subsection{The thermal components}

Here, we examine the behaviour of these thermal components in order to try and determine their origin. One subtle difference between P13 and the broader ULX population worth noting is that for the hotter of the two components we find that the data prefer a steep radial temperature index (i.e. $p \gtrsim 1.0$ ) when fit with a disc model (DISKPBB). This is true for all our various analyses of the P13 data (the time-averaged and phase-resolved analysis of epoch $\mathrm{XN1}$, and the multi-epoch analysis of epochs X2, X3 and XN1). In fact, for our multi-epoch analysis, the fit is slightly improved if we do not allow for a range of temperatures at all, and fit the hotter component as a single-temperature blackbody (Section 4.2). For other ULX systems, the radial temperature indices obtained for the hotter component have typically been much flatter, with $p<0.75$ (e.g. Walton et al. 2015b, 2017).

In Fig. 12, we plot the flux of each of the DISKBB and вв components (computed over $0.01-100 \mathrm{keV}$, a sufficiently broad-band to effectively be bolometric) against their temperatures. With only three data points to date, we do not formally fit any relations to these data, but for comparison we do overlay illustrative $F \propto T^{4}$ relations as expected for blackbody emission from a constant emitting area. The lower energy DISKBB component does appear to follow $F \propto T^{4}$ reasonably well, suggesting a roughly constant emitting area, but the same is not true for the Bв component. In the latter case, epoch XN1 sits significantly off any extrapolation of an $F \propto T^{4}$ relation from the lower flux data, suggesting the emitting area for this component may be variable.

For the blackbody component, the maximum and minimum flux/temperature combinations (from epochs $\mathrm{XN} 1$ and $\mathrm{X} 2$, respectively) imply emitting areas with radii of $\sim 40-70 \mathrm{~km}$ from standard blackbody theory, or equivalently $\sim 20-35 R_{\mathrm{G}}$ for a $1.4 \mathrm{M}_{\odot}$ neutron star (where $R_{\mathrm{G}}=G M / c^{2}$ is the gravitational radius). This is comfortably larger than typical radii for the entire neutron star $(\sim 10 \mathrm{~km})$, suggesting that this component must come from the accretion flow rather than from the surface of the neutron star itself, particularly given that the emission from the surface of a magnetized neutron star should primarily arise from small hotspots around the magnetic poles on to which the accretion is channelled. For the lower temperature DISKBB component, the fact that this component is required to have a range of temperatures already suggests that this must also be associated with the accretion flow. This is further supported by the emitting radius implied by the DISKBB normalizations, which are proportional to $R_{\text {in }}^{2} \cos \theta / f_{\text {col }}^{4}$. Here, $R_{\text {in }}$ and $\theta$ are the inner radius and the inclination of the disc, and $f_{\text {col }}$ is the colour correction factor relating the observed 'colour' temperature to the effective temperature at the midplane of the disc $\left(f_{\text {col }}=T_{\text {col }} / T_{\text {eff }}\right)$. This latter quantity provides a simple empirical correction accounting for the complex physics in the disc atmosphere. Even assuming that both $\cos \theta$ and $f_{\text {col }}$ are unity (i.e. no colour correction and a face-on disc; note that this is also implicitly assumed for the hotter thermal component in our use of standard blackbody theory above), which would give the smallest radii, we find that $R_{\mathrm{in}} \sim 280-360 \mathrm{~km}\left(\sim 140-180 R_{\mathrm{G}}\right)$. This is at least an order of magnitude larger than typical neutron star radii. We will re-visit these radii in Section 6.3.

\subsection{The pulsed emission/high-energy tail}

In addition to the two thermal components, our analysis of the time-averaged spectrum finds that a third continuum component is required above $10 \mathrm{keV}$, as all two-component thermal models leave a clear excess at the highest energies probed by NuSTAR (Fig. 1). 


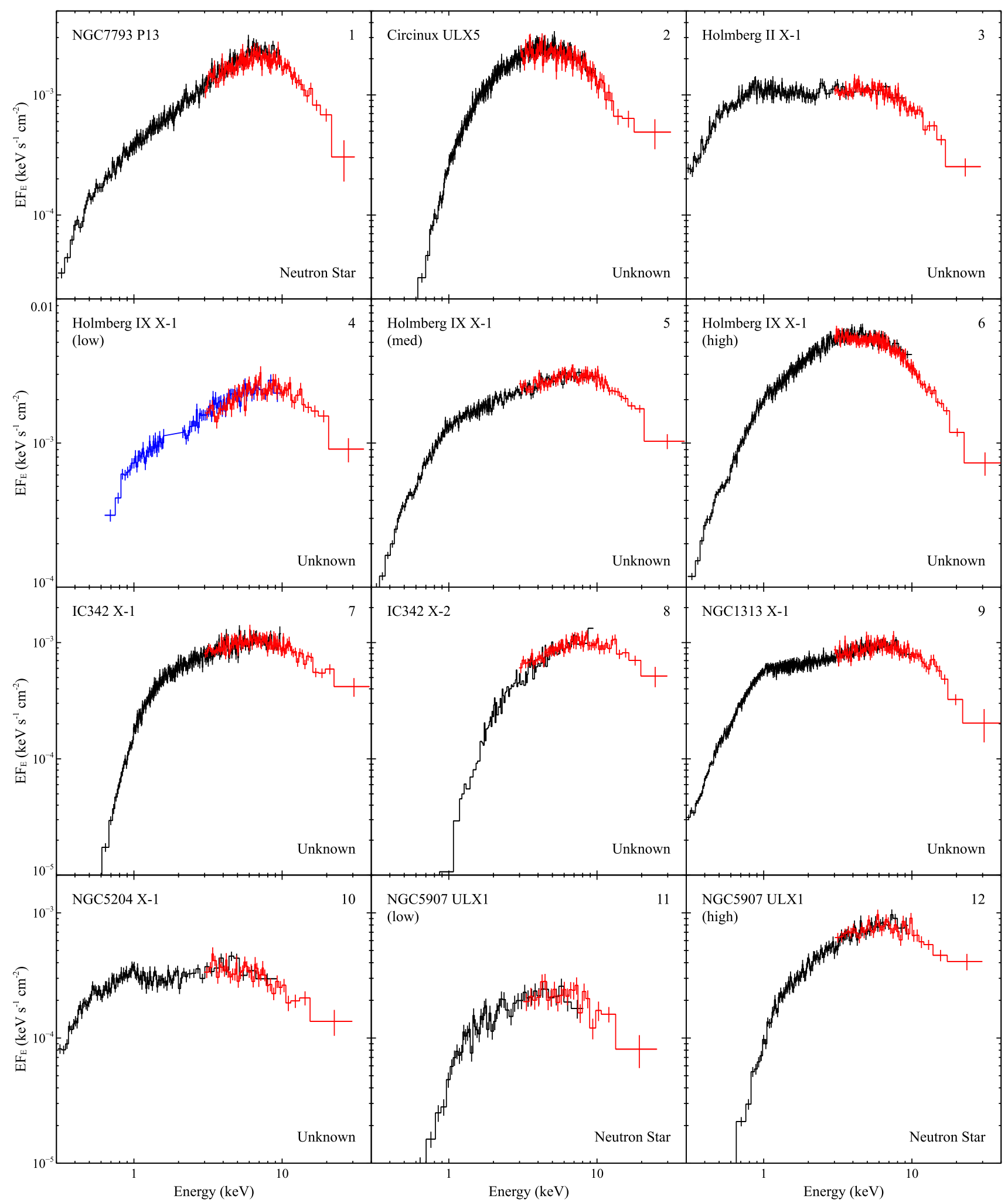

Figure 11. Broad-band spectra of the ULX population with simultaneous coverage in both soft (XMM-Newton, Suzaku) and hard (NuSTAR) X-rays; as for Fig. 1 (left), the data have been unfolded through a model simply consisting of a constant. Only sources which suffer from negligible source confusion and which are robustly detected out to at least $20 \mathrm{keV}$ are included, and for clarity, only data from the XMM-Newton EPIC-pn (black), NuSTAR FPMA (red) and Suzaku front-illuminated XIS (blue) detectors are shown (the only exception is NGC 5907 ULX, where for NuSTAR we show the combined FPMA+FPMB data owing to the lower S/N in this case). The sample includes P13 (panel 1; this work), Circinus ULX5 (panel 2; adapted from Walton et al. 2013), Holmberg II X-1 (panel 3; adapted from Walton et al. 2015b), Holmberg IX X-1 (panels 4-6; adapted from Walton et al. 2017), IC 342 X-1 and X-2 (panels 7 and 8, respectively; adapted from Rana et al. 2015), NGC 1313 X-1 (panel 9; adapted from Bachetti et al. 2013), NGC 5204 X-1 (panel 10; adapted from Mukherjee et al. 2015) and NGC 5907 ULX (panels 11-12; adapted from Fürst et al. 2017). 


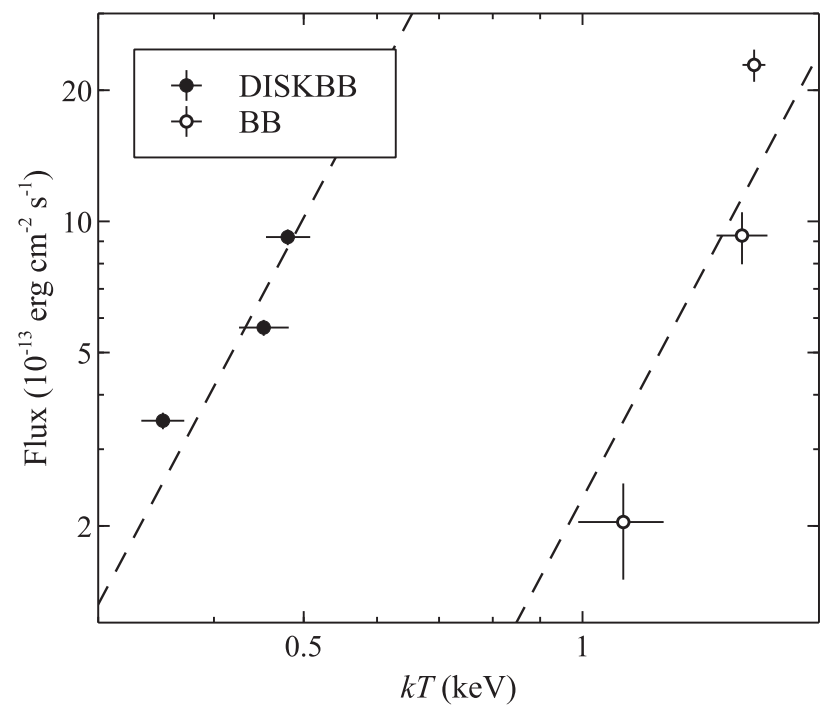

Figure 12. Flux versus temperature for both the DISKBB (filled circles) and Bв (empty circles) components in our multi-epoch spectral analysis. For illustration, we also show example $F \propto T^{4}$ relations (dashed lines).

As discussed previously, similar high-energy excesses have been seen in the average spectra of several other ULX systems now. In these previous works, the excess has typically been modelled as steep $(\Gamma \sim 3)$ high-energy power-law tail to the lower energy thermal continuum components, assumed to arise through Compton up-scattering similar to the X-ray coronae seen in sub-Eddington systems. When considering the time-averaged spectrum for P13, the same model fits the data well.

However, through phase-resolved analyses of the P13 data, we find that this excess is actually associated with the pulsed emission component arising from the magnetically channelled accretion columns. rather than from a traditional $\mathrm{X}$-ray corona. The emission from the accretion column dominates over the two thermal components above $10 \mathrm{keV}$, naturally explaining the fact that the pulsed fraction increases with increasing energy below $10 \mathrm{keV}$, and appears to saturate at higher energies (Fürst et al. 2016b). This emission is well described with a simple CUTOFFPL model, which has a very hard rise $(\Gamma \sim 0.15)$ below $10 \mathrm{keV}$ before breaking to a steep spectrum above $10 \mathrm{keV}$ thanks to a low cutoff energy $\left(E_{\mathrm{cut}} \sim 4.5 \mathrm{keV}\right)$. This is qualitatively similar to the pulsed emission from the first ULX pulsar discovered, M82 X-2 (Brightman et al. 2016a, although for M82 $\mathrm{X}-2$ the rise is not quite as hard and the cutoff at a higher energy, see Fig. 2). The average spectrum of X-2 cannot easily be disentangled from M82 X-1, complicating spectral analyses. Nevertheless, this does show that the emission from the accretion column also extends up to these energies, and the pulsed fraction for M82 X-2 is also known to increase with increasing energy (Bachetti et al. 2014), despite the confusion with $\mathrm{X}-1$. We therefore expect that the emission from the accretion column also dominates at the highest energies in this system.

\subsection{Super-Eddington accretion on to pulsar ULXs}

For super-Eddington accretion on to a compact object, the inner regions of the accretion flow are expected to have a large scaleheight, owing to the increased support by the intense radiation pressure (e.g. Shakura \& Sunyaev 1973; Abramowicz et al. 1988; Poutanen et al. 2007; Dotan \& Shaviv 2011). This occurs interior to the point at which the accretion flow is locally at the Eddington limit, a radius commonly referred to as the 'spherization radius' $\left(R_{\mathrm{sp}}\right)$. The geometry of a super-Eddington accretion flow is expected to resemble a funnel-like structure close to the central accretor that transitions to a more standard thin disc at larger radii. The central accretors powering ULXs have generally been assumed to be black holes in the literature, and theoretical effort has therefore primarily focused on super-Eddington accretion on to these objects, but King (2008) argue that the same framework should hold for super-Eddington accretion on to a neutron star (at least, in regions of the disc not significantly influenced by the neutron star's magnetic field).

In the context of this model, the two thermal components required to successfully reproduce ULX spectra below $10 \mathrm{keV}$ are often interpreted as arising from different regions in a super-Eddington accretion flow, with the hotter and cooler components arising from inside and outside the inner funnel, respectively (e.g. Middleton et al. 2015; Luangtip et al. 2016, but see Miller et al. 2014 for an alternative scenario). Large scaleheight, optically thick accretion flows are expected to show $p<0.75$ owing to the significant photon advection that should occur in this region (Abramowicz et al. 1988), as is often seen for the hotter component in ULX spectral modelling (e.g. Walton et al. 2015b, 2017), but is not seen for P13.

For ULX pulsars, the influence of the neutron star's magnetic field also needs to be considered, This will disrupt the disc at the magnetospheric radius $\left(R_{\mathrm{M}}\right)$, at which point the accreting material is forced to follow the field lines down on to the neutron star. Since the discovery of the first ULX pulsar (Bachetti et al. 2014), an extremely wide range of magnetic fields have been proposed in the literature in order to explain the observed properties of these sources, from $B \sim 10^{9-14} \mathrm{G}$ (e.g. Kluźniak \& Lasota 2015; Mushtukov et al. 2015). This naturally leads to a range of predictions for the accretion geometry, depending on the relative sizes of $R_{\mathrm{sp}}$ and $R_{\mathrm{M}}$. If $R_{\mathrm{M}}>R_{\mathrm{sp}}$ (high B-field), then the magnetic pressure will terminate the accretion flow before the large scaleheight inner funnel can form. Alternatively, if $R_{\mathrm{M}} \ll R_{\text {sp }}$ (low B-field), then the disc will only truncate very close to the neutron star, allowing the inner funnel to form unhindered, and the geometry will resemble that described above. Finally, for more intermediate configurations in which $R_{\mathrm{M}} \lesssim R_{\mathrm{sp}}$ (i.e. smaller, but still roughly similar), then the inner funnel should begin to form, but will be terminated prematurely by the magnetic field.

The fact that even after accounting for the pulsed emission from the accretion column we still require two thermal components to reproduce the P13 data supports the idea that there are two distinct regions to the accretion flow beyond $R_{\mathrm{M}}$, suggesting that $R_{\mathrm{M}}<R_{\mathrm{sp}}$. One might therefore expect to be able to associate the characteristic radii of the hotter and cooler components with $R_{\mathrm{M}}$ and $R_{\mathrm{sp}}$, respectively. Our previous crude estimates of these radii are qualitatively consistent with this scenario, with the hotter component arising from a smaller region. Interestingly, the radii of these two components appear to be roughly comparable (differing by a factor of $\sim 6$; Section 6.1).

Several additional factors need to be considered to assess whether these radii could really be similar in reality. The values presented in Section 6.1 were estimated by setting both $f_{\text {col }}$ and $\cos \theta$ to unity in order to assess whether either component could be associated with the neutron star surface. Both of these quantities can have a strong influence on the absolute radii inferred. However, as long as $f_{\text {col }}$ and $\theta$ are relatively similar for both components, their relative radii should be fairly insensitive to the values adopted. On some level, we might expect that $f_{\text {col }}$ should be larger for the hotter component (Shimura \& Takahara 1995), which would increase the radius of this component relative to the cooler one. Large differences in $f_{\mathrm{col}}$ 
are not likely though (Shimura \& Takahara 1995; Davis, Done \& Blaes 2006), so we expect this will only influence the relative sizes inferred by up to a factor of a few. In addition, if the вв component does arise from the regions of the flow interior to $R_{\mathrm{sp}}$, then this will likely be subject to some level of geometric collimation by the inner funnel. As such, the observed flux will be enhanced relative to the intrinsic flux by a beaming factor $b$ (i.e. $L_{\text {int }}=b L_{\text {obs }}$ adopting the nomenclature of King 2008, where $b \leq 1$, and $b=1$ represents isotropic emission). Since the outer disc would be largely immune to similar geometric beaming this would serve to decrease the radius of the hotter component relative to the cooler one, but only by a factor of $b^{-1 / 2}$. For illustration, the beaming factor of $b \sim 0.1$ suggested by King (2008) for neutron star accretors reaching $L_{X} \sim 10^{40} \mathrm{erg} \mathrm{s}^{-1}$ (similar to P13) would only change the relative radii of the thermal components by a factor of a few. Furthermore, truncation of the disc by the magnetic field will reduce the degree of geometric beaming relative to the case in which the magnetic field has a negligible effect on the flow (as assumed by King 2008). Given that these two issues effects act in opposition to each other, and may result in corrections of a similar order, the conclusion that $R_{\mathrm{sp}}$ and $R_{\mathrm{M}}$ are comparable would still appear to hold.

If $R_{\mathrm{M}}$ is fairly similar to $R_{\mathrm{sp}}$, then this could potentially explain why the hotter thermal component in P13 does not require a strong range of temperatures. If the magnetic field quickly truncates the disc for $R<R_{\mathrm{sp}}$, then the geometrically thick inner region of the flow would only exist over a fairly narrow range of radii, and subsequently only emit over a narrow range of temperatures. The preference for a single blackbody/steep radial temperature index is likely driven in part by the assumption that the disc extends out to radii $\gg R_{\text {in }}$ (taken to be $R_{\mathrm{M}}$ in our simple picture), which is implicit in the DISKPBB model. This could artificially force $p$ to be steep in the fits since this is the only way to make the DISKPBB model effectively emit over a narrow range of temperatures, even if in reality $p<0.75$ between $R_{\mathrm{sp}}$ and $R_{\mathrm{M}}$, as would be expected for a thick super-Eddington disc.

It is interesting to note that King, Lasota \& Kluźniak (2017) also suggest that $R_{\mathrm{sp}}$ and $R_{\mathrm{M}}$ are similar for the three ULX pulsars identified to date. However, we caution that this is based on a model which assumes that the lower temperature component represents the blackbody emission from within the inner funnel of a superEddington flow. Furthermore, the model is designed to reproduce a luminosity-temperature relation of the form $L \propto T^{-4}$ (note the sign on the exponent), which has previously been claimed observationally for this component. This is primarily based on the work of Kajava \& Poutanen (2009), who find an anti-correlation between luminosity and temperature with this approximate form by fitting the results for the soft thermal component compiled from a sample of ULXs. More recent analyses do not support such a relation for the soft component when individual sources are considered (Miller et al. 2013), instead finding positive indices (i.e. luminosity positively correlated with temperature). Critically, for P13 we find that both the cooler DISKBB and the hotter blackbody components show positive correlations between luminosity and temperature, and certainly do not follow $L \propto T^{-4}$. Nevertheless, our results would appear broadly consistent with the broad conclusions of King et al. (2017) with regard to the similarity of $R_{\mathrm{sp}}$ and $R_{\mathrm{M}}$.

Although it is challenging to constrain the absolute value of $R_{\mathrm{M}}$ for P13 from our analysis given the issues related to beaming and the colour correction discussed above, if $R_{\mathrm{M}}<R_{\mathrm{sp}}$ (as is supported by the requirement for two non-pulsing blackbody components) then we can obtain a rough upper limit on the magnetic field of the neutron star by taking the limit that $R_{\mathrm{M}}=R_{\mathrm{sp}}$. For a dipole field and a typical neutron star mass and radius, the magnetospheric radius is given by $R_{\mathrm{M}} \sim\left(2.6 \times 10^{8}\right) \dot{M}_{17}^{-2 / 7} B_{12}^{4 / 7} \mathrm{~cm}$ (Lamb, Pethick \& Pines 1973; Cui 1997; Fuerst et al. 2017), where $\dot{M}_{17}$ is the accretion rate at $R_{\mathrm{M}}$ in units of $10^{17} \mathrm{~g} \mathrm{~s}^{-1}$ and $B_{12}$ is the magnetic field strength in units of $10^{12} \mathrm{G}$. Standard super-Eddington theory suggests the location of $R_{\mathrm{sp}}$ is related to the input accretion rate: $R_{\mathrm{sp}} / R_{\mathrm{G}} \sim$ (27/4) $\dot{m}_{0}$ (independent of the nature/magnetic configuration of the central accretor), where $\dot{m}_{0}$ is the input accretion rate in Eddington units (Shakura \& Sunyaev 1973), so taking this limit allows us to estimate both $R_{\mathrm{M}}$ and $\dot{M}_{17}$, assuming conservatively that the accretion rate at both $R_{\mathrm{sp}}$ and $R_{\mathrm{M}}$ is the same as $\dot{m}_{0}$ (in reality, we expect there to be strong mass-loss from the system; this should primarily occur interior to $R_{\mathrm{sp}}$, being the point at which the accretion flow becomes locally Eddington, and will reduce the accretion rate at $R_{\mathrm{M}}$ relative to that at $R_{\mathrm{sp}}$, which further emphasizes that our calculation is an upper limit).

Our prior estimate of $R_{\mathrm{sp}}$ (Section 6.1) assumed both $\cos \theta$ and $f_{\text {col }}=1$, to conservatively compare with neutron star radii. The former may be reasonable, since we appear to have an unobscured view of the inner regions of the flow (Sutton, Roberts \& Middleton 2013; Middleton et al. 2015), but the colour correction for a standard disc (expected beyond $R_{\mathrm{sp}}$ ) is more typically expected to be $f_{\text {col }} \sim 1.7$ (Shimura \& Takahara 1995). Adopting this value for $f_{\text {col }}$ increases the estimate for $R_{\mathrm{sp}}$ to $\sim 900 \mathrm{~km}$, or equivalently $R_{\mathrm{sp}} / R_{\mathrm{G}} \sim 450$, and therefore $\dot{m}_{0} \sim 66$. For a standard $1.4 \mathrm{M}_{\odot}$ neutron star the Eddington accretion rate is $\dot{M}_{\mathrm{E}}=2.2 \times 10^{18} \mathrm{~g} \mathrm{~s}^{-1}$ (assuming a radiative efficiency of $\eta \sim 0.09$ ), so the accretion rate at $R_{\mathrm{M}}$ would be $\dot{M} \sim 1.4 \times 10^{20} \mathrm{~g} \mathrm{~s}^{-1}$. Substituting all this back into the original expression for $R_{\mathrm{M}}$ and rearranging, we therefore find that $B_{12} \lesssim 6$. Interestingly, the co-rotation radius for P13 is also $R_{\mathrm{co}} \sim 900 \mathrm{~km}$, so we have $R_{\mathrm{sp}} \sim R_{\mathrm{co}}$. Since we know that $R_{\mathrm{M}}<R_{\mathrm{co}}$, as accretion must be occurring in P13, this constraint would also imply $B_{12} \lesssim 6$. Although we expect the similarity of $R_{\mathrm{sp}}$ and $R_{\mathrm{co}}$ is coincidental (as long as $R_{\mathrm{M}}<R_{\text {co }}$ there is no reason $R_{\text {co }}$ should be an important radius in terms of the observed emission), this does provide further support for our conclusion that $R_{\mathrm{M}}<R_{\mathrm{sp}}$, and that the thick inner disc does form over some range of radii.

This is consistent with the estimate in Fürst et al. (2016b), who found $B_{12} \sim 1.5$ based on the observed spin-up rate of the neutron star. However, we stress that our calculation fundamentally depends on the association of the characteristic radius of the cooler thermal component with $R_{\mathrm{sp}}$. Furthermore, since $B \propto R_{\mathrm{M}}^{7 / 4}$, any errors in the radius will be magnified in the magnetic field limit estimated here. Our limit on $B$ therefore depends very strongly on the value assumed for the colour correction factor, since the radii of the thermal components are themselves proportional to $f_{\mathrm{col}}^{2}$. We also stress that $R_{\mathrm{M}}$ is most sensitive to the dipolar field, as any quadrupolar component will decay much more rapidly with radius, so these constraints should be considered on the dipolar component only. A stronger quadrupolar component to the magnetic field that only acts close to the neutron star, as suggested by Israel et al. (2017a), cannot be excluded by these considerations.

One of the basic predictions of super-Eddington accretion is that powerful winds should be launched from the radiation-pressure dominated regions of the flow (i.e. primarily interior to $R_{\mathrm{sp}}$; e.g. Poutanen et al. 2007). Indeed, unambiguous evidence for powerful outflows has now been seen through resolved, blueshifted atomic features in a handful of other ULX systems with deep XMM-Newton coverage, primarily in the soft X-ray band $(<2 \mathrm{keV})$ but in the case of NGC $1313 \mathrm{X}-1$ also in the iron $\mathrm{K} \alpha$ band (Pinto, Middleton \& Fabian 2016; Walton et al. 2016a; Pinto et al. 2017). If our proposed scenario for P13 is correct, and thick inner regions of the 
flow do begin to form, then we might expect to see evidence for a similar wind in P13, either through ionized absorption features if the outflow crosses our line of sight, or ionized emission otherwise. Given the low levels of neutral absorption here, this could be tested with future deep observations combining either XMM-Newton or XARM (the Hitomi replacement mission; Takahashi et al. 2012) together with $N u S T A R$.

\subsection{The low-flux state}

In addition to the bright state observations, we also investigate the spectrum obtained during the off-state seen between 2011 and 2013 (epochs C2, C3, C4 and X1). The spectrum during these epochs is still very hard, and remains variable (Fig. 5). However, simple power-law fits leave clear line-like residuals at $\sim 1 \mathrm{keV}$, and the fit is significantly improved with the addition of a plasma component, which we argue is associated with the P13 system, rather than galactic-scale plasma emission (e.g. star formation). If P13 does launch a strong wind while at ULX luminosities, this plasma component could potentially arise from collisionally ionized material previously ejected from the accretion flow that becomes visible with the extreme drop in flux from the central accretor.

The hard $(\Gamma \sim 0.7)$ and variable power-law emission almost certainly suggests that there is still some residual accretion on to the neutron star during these off-states. After the discovery of pulsations from M82 X-2, Tsygankov et al. (2016) suggested that the lowflux states seen in this source could be related to the onset of the propeller effect. This occurs when $R_{\mathrm{M}}$ moves outside $R_{\mathrm{co}}$, such that the magnetic field ends up acting as a barrier to further accretion, rather than channelling the material on to the neutron star poles. As such, for $R_{\mathrm{M}}>R_{\mathrm{co}}$ the accretion rate, and therefore the observed $\mathrm{X}$-ray luminosity, suddenly drops precipitously. We do not see any clear evidence for an accretion disc component in these data; if we replace the MEKAL component with DISKBB, the fit is better than the simple power-law model $\left(\chi^{2} / \mathrm{DoF}=44 / 27\right)$, but not as good as the MEKAL model, and the line-like residuals at $\sim 1 \mathrm{keV}$ remain. This could suggest the disc would need to be truncated at a sufficiently large radius that it makes a negligible contribution to the observed flux in the X-ray band.

Nevertheless, the residual accretion flux is still quite substantial, with $L_{\mathrm{X}} \sim(3-6) \times 10^{37} \mathrm{erg} \mathrm{s}^{-1}$ in the $0.3-10.0 \mathrm{keV}$ band, i.e. $\sim 0.15-0.3 L_{\mathrm{E}}$ for a $1.4 \mathrm{M}_{\odot}$ neutron star. This is quite similar to $\mathrm{M} 82 \mathrm{X}-2$, which still radiates at $\sim 10^{38} \mathrm{erg} \mathrm{s}^{-1}$ in its off-state (Brightman et al. 2016a). As noted by Tsygankov et al. (2016), this only requires a few per cent of the accreting matter to leak through the magnetosphere, which may not be unreasonable. However, there is now evidence that the low-flux periods in M82 X-2 are related to its $\sim 62 \mathrm{~d}$ superorbital cycle (Brightman et al., submitted), which would call into question the propeller interpretation for that system. If confirmed, this would establish a significant difference between M82 X-2 and both NGC 5907 ULX and P13, as the timing and duration of the off-states seen in the latter two means they cannot be related to their similar $\sim 64$ and $\sim 78 \mathrm{~d}$ periods. This means that there may not be a single physical origin for these off-states common to all three systems, despite the changes in observed flux being similar in all three cases, and so the propeller effect cannot be excluded for the low-flux states in P13.

\subsection{Implications for the ULX population}

The qualitative similarity of the broad-band spectrum of P13 to the rest of the ULX population has significant implications. Since P13 is a known super-Eddington accretor, the XMM-Newton+NuSTAR observation of this source presented here robustly demonstrates that these spectra really are associated with super-Eddington accretion. This confirms the conclusions of our previous work on broad-band observations of ULXs (Bachetti et al. 2013; Walton et al. 2014, 2015b, 2017; Mukherjee et al. 2015; Rana et al. 2015), which were based on the lack of similarity between ULX spectra and the standard modes of sub-Eddington accretion on to black holes (see Remillard \& McClintock 2006 for a review), rather than a well-established knowledge of the spectral appearance of superEddington accretion at ULX luminosities.

The similarity between P13 and the broader ULX population may even suggest that more of these sources could be powered by accreting neutron stars. Although pulsations have not currently been observed from any other sources (Doroshenko, Santangelo \& Ducci 2015), for M82 X-2 the pulsations are known to be transient (Bachetti et al. 2014), and this also appears to be the case for NGC 5907 ULX (Israel et al. 2017a). Pintore et al. (2017) also suggest that additional ULXs could be pulsar systems, arguing that ULX spectra are well fit by spectral models typically applied to sub-Eddington pulsars: a low-energy blackbody (peaking below $2 \mathrm{keV}$ ) and a power law with an exponential cutoff produced by an accretion column that dominates at higher energies $(>2 \mathrm{keV})$. Our work suggests that the spectral decomposition used by Pintore et al. (2017) is not actually correct, and that the situation in P13 is more complex as the $2-10 \mathrm{keV}$ emission does not exclusively arise from the column. Instead, we find that this only reliably dominates the emission above $10 \mathrm{keV}$, resulting in the high-energy excess seen with models that only invoke two continuum components (Fig. 1). It is possible therefore that the presence of such a hard excess is indicative that the accretion in the innermost regions occurs via magnetically channelled columns, rather than via a disc. We stress again that a number of other ULXs have now been seen to exhibit similar excesses above $\sim 10 \mathrm{keV}$, so if this is correct then neutron stars/pulsars could potentially be prevalent in the ULX population.

Since the pulsations are seen to be transient in $2 / 3$ of the known ULX pulsar systems, additional means of firmly identifying pulsar/neutron star accretors may well be the key to expanding this population. Indeed, King et al. (2017) suggest that pulsations may only be observable from neutron star ULXs when $R_{\mathrm{sp}}$ and $R_{\mathrm{M}}$ are similar (as we find appears to be the case for P13), as they argue that for $R_{\mathrm{M}} \ll R_{\mathrm{sp}}$ the pulsations could be diluted to the point of being undetectable by the non-pulsed emission from the disc. Although our work finds that the highest energies ( $\gtrsim 15 \mathrm{keV}$ ) should be relatively immune to such issues as the column always dominates, the low count rates observed at these energies would still serve as a major barrier to the detection of pulsations, further emphasizing the need for additional means of identification.

One alternative method may be to search for other sources that show similar off-states to those seen from all three ULX pulsars, as suggested by Fürst et al. (2016a). In addition to this, based on our analysis of P13 in this work we tentatively suggest that finding that the hotter thermal component does not require a run of temperatures may be another indicator of a neutron star accretor. This would provide an indication that the disc is truncated close to $R_{\mathrm{sp}}$, despite the source maintaining ULX luminosities, and would in turn imply a magnetized central accretor.

\section{SUMMARY AND CONCLUSIONS}

We have undertaken a detailed X-ray spectral analysis of the available data for P13 - a key laboratory for ULX pulsars owing to its 
lack of source confusion, persistently detected pulsations and low obscuration - focusing on the first high-energy detection of this source with NuSTAR. Through time-averaged, phase-resolved and multi-epoch studies, we find that two thermal components are required to fit the data below $10 \mathrm{keV}$, in addition to a third continuum component above $10 \mathrm{keV}$ to account for the high-energy excess seen with dual-thermal models. This is qualitatively very similar to the rest of the ULX population with broad-band coverage, for which the nature of the accretors generally remain unknown, confirming the super-Eddington nature of these sources and suggesting that additional ULXs may be neutron stars/pulsars. Our phase-resolved analysis finds that the third, high-energy component is associated with the magnetically collimated accretion columns that must be present in P13 in order to produce the observed pulsations. Neither of the two thermal components can arise from the neutron star itself, as their characteristic radii at least an order of magnitude too large, suggesting that they both arise from the accretion flow. In turn, this suggests that the accretion flow has two distinct regions, as expected for super-Eddington accretion flows which should transition from a standard thin outer disc to a geometrically thick inner disc at the point that the flow reaches the local Eddington limit $\left(R_{\mathrm{sp}}\right)$. However, the radii for these two components appear to be comparable, and the hotter component is better described as a single blackbody than a thick, advection-dominated accretion disc, which should have a strong run of temperatures.

We suggest that P13 may represent a scenario in which the magnetospheric radius - the point at which the disc is truncated and the accreting material is forced along the magnetic field lines - is smaller than, but still similar to $R_{\mathrm{sp}}$. This would allow the thicker inner regions of the super-Eddington flow to begin to develop, before being truncated prematurely by the magnetic field. Such a scenario would simultaneously explain the need for two thermal components, their fairly similar radii, and the fact that the hotter component does not need a run of temperatures, as the thick inner disc would only persist over a fairly narrow range of radii, and thus only emit over a fairly narrow range of temperatures. This similarity would imply an upper limit to the neutron stars dipolar magnetic field of $B \lesssim$ $6 \times 10^{12} \mathrm{G}$, albeit with large uncertainties. If this is correct, evidence of similar truncation of the disc in other sources could offer a means of identifying additional neutron star/pulsar ULXs, as this would not be expected for black hole systems.

Finally, P13 is known to exhibit unusual 'off' states in which the X-ray flux drops by many orders of magnitude, potentially related to the propeller effect. We also examine the spectra obtained during one of these events, and find that the data require both a hard power-law component, suggesting residual accretion on to the neutron star, and emission from a thermal plasma, which we argue is likely associated with the P13 system.

\section{ACKNOWLEDGEMENTS}

The authors would like to thank the reviewer for the feedback provided, which helped to improve the final version of the manuscript. DJW and MJM acknowledge support from an STFC Ernest Rutherford fellowship, ACF acknowledges support from ERC Advanced Grant 340442 and DB acknowledges financial support from the French Space Agency (CNES). This research has made use of data obtained with NuSTAR, a project led by Caltech, funded by NASA and managed by NASA/JPL, and has utilized the NUSTARDAS software package, jointly developed by the ASDC (Italy) and Caltech (USA). This work has also made use of data obtained with XMM-
Newton, an ESA science mission with instruments and contributions directly funded by ESA Member States.

\section{REFERENCES}

Abramowicz M. A., Czerny B., Lasota J. P., Szuszkiewicz E., 1988, ApJ, 332,646

Annuar A. et al., 2015, ApJ, 815, 36

Arnaud K. A., 1996, in Jacoby G. H., Barnes J., eds, ASP Conf. Ser., Vol. 101, Astronomical Data Analysis Software and Systems V. Astron. Soc. Pac., San Francisco, p. 17

Bachetti M. et al., 2013, ApJ, 778, 163

Bachetti M. et al., 2014, Nature, 514, 202

Basko M. M., Sunyaev R. A., 1976, MNRAS, 175, 395

Brightman M. et al., 2016a, ApJ, 816, 60

Brightman M. et al., 2016b, ApJ, 829, 28

Cash W., 1979, ApJ, 228, 939

Coburn W., Heindl W. A., Rothschild R. E., Gruber D. E., Kreykenbohm I., Wilms J., Kretschmar P., Staubert R., 2002, ApJ, 580, 394

Cui W., 1997, ApJ, 482, L163

Dall'Osso S., Perna R., Stella L., 2015, MNRAS, 449, 2144

Davis S. W., Done C., Blaes O. M., 2006, ApJ, 647, 525

Doroshenko V., Santangelo A., Ducci L., 2015, A\&A, 579, A22

Dotan C., Shaviv N. J., 2011, MNRAS, 413, 1623

Earnshaw H. M. et al., 2016, MNRAS, 456, 3840

Ekşi K. Y., Andaç d. C., Çıkıntoğlu S., Gençali A. A., Güngör C., Öztekin F., 2015, MNRAS, 448, L40

Fuerst F. et al., 2017, A\&A, 606, 89

Fürst F. et al., 2013, ApJ, 779, 69

Fürst F. et al., 2014, ApJ, 784, L40

Fürst F. et al., 2016a, ApJ, 819, 150

Fürst F. et al., 2016b, ApJ, 831, L14

Fürst F., Walton D. J., Stern D., Bachetti M., Barret D., Brightman M., Harrison F. A., Rana V., 2017, ApJ, 834, 77

Garmire G. P., Bautz M. W., Ford P. G., Nousek J. A., Ricker G. R., Jr, 2003, in Truemper J. E., Tananbaum H. D., eds, Proc. SPIE Conf. Ser. Vol. 4851, X-Ray and Gamma-Ray Telescopes and Instruments for Astronomy. SPIE, Bellingham, p. 28

Gehrels N. et al., 2004, ApJ, 611, 1005

Gladstone J. C., Roberts T. P., Done C., 2009, MNRAS, 397, 1836

Harrison F. A. et al., 2013, ApJ, 770, 103

Herold H., 1979, Phys. Rev. D, 19, 2868

Hu C.-P., Li K. L., Kong A. K. H., Ng C.-Y., Lin L. C.-C., 2017, ApJ, 835, L9

Islam N., Maitra C., Pradhan P., Paul B., 2015, MNRAS, 446, 4148

Israel G. L. et al., 2017a, Science, 355, 817

Israel G. L. et al., 2017b, MNRAS, 466, L48

Jansen F. et al., 2001, A\&A, 365, L1

Kajava J. J. E., Poutanen J., 2009, MNRAS, 398, 1450

Kalberla P. M. W., Burton W. B., Hartmann D., Arnal E. M., Bajaja E., Morras R., P"oppel W. G. L., 2005, A\&A, 440, 775

King A. R., 2008, MNRAS, 385, L113

King A., Lasota J.-P., 2016, MNRAS, 458, L10

King A., Lasota J.-P., Kluźniak W., 2017, MNRAS, 468, L59

Kluźniak W., Lasota J.-P., 2015, MNRAS, 448, L43

Kong A. K. H., Hu C.-P., Lin L. C.-C., Li K. L., Jin R., Liu C. Y., Yen D. C.-C., 2016, MNRAS, 461, 4395

Krivonos R., Sazonov S., 2016, MNRAS, 463, 756

Lamb F. K., Pethick C. J., Pines D., 1973, ApJ, 184, 271

Luangtip W., Roberts T. P., Done C., 2016, MNRAS

Madsen K. K. et al., 2015, ApJS, 220, 8

Middleton M. J., Heil L., Pintore F., Walton D. J., Roberts T. P., 2015, MNRAS, 447, 3243

Miller J. M., Walton D. J., King A. L., Reynolds M. T., Fabian A. C., Miller M. C., Reis R. C., 2013, ApJ, 776, L36

Miller J. M., Bachetti M., Barret D., Harrison F. A., Fabian A. C., Webb N. A., Walton D. J., Rana V., 2014, ApJ, 785, L7 
Mineshige S., Hirano A., Kitamoto S., Yamada T. T., Fukue J., 1994, ApJ, 426, 308

Mitsuda K. et al., 1984, PASJ, 36, 741

Motch C., Pakull M. W., Soria R., Grisé F., Pietrzyński G., 2014, Nature, 514,198

Mukherjee E. S. et al., 2015, ApJ, 808, 64

Mushtukov A. A., Suleimanov V. F., Tsygankov S. S., Poutanen J., 2015, MNRAS, 454, 2539

Odaka H., Khangulyan D., Tanaka Y. T., Watanabe S., Takahashi T., Makishima K., 2013, ApJ, 767, 70

Pietrzyński G. et al., 2010, AJ, 140, 1475

Pinto C., Middleton M. J., Fabian A. C., 2016, Nature, 533, 64

Pinto C. et al., 2017, MNRAS, 468, 2865

Pintore F., Zampieri L., Stella L., Wolter A., Mereghetti S., Israel G. L., 2017, ApJ, 836, 113

Poutanen J., Lipunova G., Fabrika S., Butkevich A. G., Abolmasov P., 2007, MNRAS, 377, 1187

Protassov R., van Dyk D. A., Connors A., Kashyap V. L., Siemiginowska A., 2002, ApJ, 571, 545

Rana V. et al., 2015, ApJ, 799, 121

Remillard R. A., McClintock J. E., 2006, ARA\&A, 44, 49

Shakura N. I., Sunyaev R. A., 1973, A\&A, 24, 337

Shidatsu M., Ueda Y., Fabrika S., 2017, ApJ, 839, 46

Shimura T., Takahara F., 1995, ApJ, 445, 780

Steiner J. F., Narayan R., McClintock J. E., Ebisawa K., 2009, PASP, 121, 1279

Stobbart A.-M., Roberts T. P., Wilms J., 2006, MNRAS, 368, 397

Strüder L. et al., 2001, A\&A, 365, L18
Sutton A. D., Roberts T. P., Middleton M. J., 2013, MNRAS, 435, 1758

Takahashi T. et al., 2012, Proc. SPIE Conf. Ser. Vol. 8443, Space Telescopes and Instrumentation 2012: Ultraviolet to Gamma Ray. SPIE, Bellingham, p. $84431 \mathrm{Z}$

Titarchuk L., 1994, ApJ, 434, 570

Tsygankov S. S., Mushtukov A. A., Suleimanov V. F., Poutanen J., 2016, MNRAS, 457, 1101

Tully R. B., Courtois H. M., Sorce J. G., 2016, AJ, 152, 50

Turner M. J. L. et al., 2001, A\&A, 365, L27

Verner D. A., Ferland G. J., Korista K. T., Yakovlev D. G., 1996, ApJ, 465, 487

Vybornov V., Klochkov D., Gornostaev M., Postnov K., Sokolova-Lapa E., Staubert R., Pottschmidt K., Santangelo A., 2017, A\&A, 601, A126

Walton D. J. et al., 2013, ApJ, 779, 148

Walton D. J. et al., 2014, ApJ, 793, 21

Walton D. J. et al., 2015a, ApJ, 799, 122

Walton D. J. et al., 2015b, ApJ, 806, 65

Walton D. J. et al., 2016a, ApJ, 826, L26

Walton D. J. et al., 2016b, ApJ, 826, 87

Walton D. J. et al., 2016c, ApJ, 827, L13

Walton D. J. et al., 2017, ApJ, 839, 105

Weisskopf M. C., Brinkman B., Canizares C., Garmire G., Murray S., Van Speybroeck L. P., 2002, PASP, 114, 1

Wilms J., Allen A., McCray R., 2000, ApJ, 542, 914

This paper has been typeset from a $\mathrm{T}_{\mathrm{E}} \mathrm{X} / \mathrm{L}_{\mathrm{E}} \mathrm{X}$ file prepared by the author. 